\title{
Parabolic, hyperbolic and elliptic Poincaré series
}

\author{
by

\section{Özlem ImamoḠLu (Zürich) and Cormac O'Sullivan (New York)}

1. Introduction. Let $\Gamma \subseteq \mathrm{PSL}_{2}(\mathbb{R})$ be a Fuchsian group of the first kind acting on the upper half-plane $\mathbb{H}$. We write $x+i y=z \in \mathbb{H}$ and set $d \mu z$ to be the $\mathrm{SL}_{2}(\mathbb{R})$-invariant hyperbolic volume form $d x d y / y^{2}$. Assume the volume of the quotient space $\Gamma \backslash \mathbb{H}$ is equal to $V<\infty$. Let $S_{k}(\Gamma)$ be the space of holomorphic weight $k$ cusp forms for $\Gamma$. This is the vector space of holomorphic functions $f$ on $\mathbb{H}$ which decay rapidly in each cusp of $\Gamma$ and satisfy the transformation property

$$
\frac{f(\gamma z)}{j(\gamma, z)^{k}}-f(z)=0 \quad \text { for all } \gamma \in \Gamma,
$$

with $j\left(\left(\begin{array}{ll}a & b \\ c & d\end{array}\right), z\right)=c z+d$ for $\gamma=\left(\begin{array}{ll}a & b \\ c & d\end{array}\right)$. We do not assume that $\Gamma$ has cusps. If there are none then we may ignore the rapid decay condition for $S_{k}$. We assume throughout that the multiplier system is trivial and that, unless otherwise stated, $4 \leq k \in 2 \mathbb{Z}$. Using the notation $\left(\left.f\right|_{k} \gamma\right)(z):=f(\gamma z) / j(\gamma, z)^{k}$, extended to all $\mathbb{C}\left[\mathrm{PSL}_{2}(\mathbb{R})\right]$ or $\mathbb{C}\left[\mathrm{SL}_{2}(\mathbb{R})\right]$ by linearity, (1.1) can be written more simply as $\left.f\right|_{k}(\gamma-1)=0$.

The automorphy property (1.1) may be modified to obtain different families of objects: for example mixed cusp forms [21] or vector-valued cusp forms [18]. In this article we generalize (1.1) to define higher-order automorphic forms. These have arisen independently in different contexts (see for example $[7,8,17])$. They are also an interesting special case of the vector-valued cusp forms of Knopp and Mason [18, 22] corresponding to unipotent representations of $\Gamma$. See [14, Theorem 2.1] for an explicit example of this connection in the context of higher-order non-holomorphic Eisenstein series. The first historical appearance of second-order forms appears to be in a paper by Eichler [6, Section 4] where he defines the second-order parabolic Poincaré series in connection with his work on automorphic integrals and their periods; see Section 8.2 below. 
Let $\mathbb{N}$ be the natural numbers $\{1,2,3, \ldots\}$ and $\mathbb{N}_{0}=\mathbb{N} \cup\{0\}$.

Definition 1. For $n \in \mathbb{N}_{0}$, the $\mathbb{C}$-vector space $S_{k}^{n}(\Gamma)$ is defined recursively as follows. Let $S_{k}^{0}(\Gamma)$ consist only of the function $\mathbb{H} \rightarrow 0$. For $n \geq 1$, let $S_{k}^{n}(\Gamma)$ contain all holomorphic functions $f: \mathbb{H} \rightarrow \mathbb{C}$ that satisfy

$$
\left.f\right|_{k}(\gamma-1) \in S_{k}^{n-1}(\Gamma) \quad \text { for all } \gamma \in \Gamma \text {. }
$$

For all parabolic elements $\pi$ of $\Gamma$ we also require

$$
\left.f\right|_{k}(\pi-1)=0 .
$$

Finally, $f$ must decay rapidly in each cusp (this is explained in Section 2.1).

We call $S_{k}^{n}(\Gamma)$ the space of order $n$, weight $k$ holomorphic cusp forms for $\Gamma$. Usually, we just write $S_{k}^{n}$. Clearly, $S_{k}^{1}$ and $S_{k}$ are synonymous. Induction, as in [14, Lemma 3.1], shows $S_{k}^{n_{1}} \subseteq S_{k}^{n_{2}}$ for any two integers $0 \leq n_{1} \leq n_{2}$. Therefore $S_{k} \subseteq S_{k}^{n}$ and higher-order forms are a generalization of the usual cusp forms.

The identity in $\Gamma$ is $I= \pm\left(\begin{array}{ll}1 & 0 \\ 0 & 1\end{array}\right)$. The remaining elements may be partitioned into three sets: the parabolic, hyperbolic and elliptic elements. These correspond to translations, dilations and rotations, respectively, in $\mathbb{H}$. As is well-known, the relation (1.1) for parabolic elements leads to a Fourier expansion of $f$ associated to each cusp of $\Gamma$. The parabolic Fourier coefficients that arise often contain a great deal of number-theoretic information. A family of corresponding parabolic Poincaré series can be constructed whose inner products with $f$ produce these Fourier coefficients.

Much less well-known are Petersson's hyperbolic and elliptic Fourier expansions, introduced in [24]. In the first half of this paper we start by giving an exposition of Petersson's work. For the benefit of the reader we develop all three expansions and their associated Poincaré series in Sections 2-4. We give a unified treatment with notation that emphasizes the similarities of the three cases.

The series we construct in Sections 2-4 are all examples of relative Poincaré series.

THEOREM 1. Let $\Gamma_{0}$ be a subgroup of $\Gamma$ and $\phi$ a holomorphic function on $\mathbb{H}$ satisfying $\left.\phi\right|_{k} \gamma=\phi$ for all $\gamma$ in $\Gamma_{0}$ and

$$
\int_{\Gamma_{0} \backslash \mathbb{H}}|\phi(z)| y^{k / 2} d \mu z<\infty .
$$

Then the relative Poincaré series

$$
P[\phi](z):=\sum_{\gamma \in \Gamma_{0} \backslash \Gamma}\left(\left.\phi\right|_{k} \gamma\right)(z)
$$

converges absolutely and uniformly on compact subsets of $\mathbb{H}$ to an element of $S_{k}$. 
This theorem is stated in [15] and proved in [20, Chapter 1, §7], for example. Variations of it also appear explicitly and implicitly in many other works. The proof given in Section 5 simply involves showing that the series (1.5) is bounded by the integral (1.4).

In the second half of the paper we show how these ideas extend naturally to the second-order space $S_{k}^{2}$. In Section 8 we prove the analog of Theorem 1 . Let $\operatorname{Hom}_{0}(\Gamma, \mathbb{C})$ be the homomorphisms from $\Gamma$ to $\mathbb{C}$ that are 0 on the parabolic elements of $\Gamma$. The following relative Poincaré series, twisted by such a homomorphism, are second-order forms.

THEOREM 2. Let $\Gamma_{0}$ be a subgroup of $\Gamma$ and $\phi$ a holomorphic function on $\mathbb{H}$ satisfying $\left.\phi\right|_{k} \gamma=\phi$ for all $\gamma$ in $\Gamma_{0}$. Let $L \in \operatorname{Hom}_{0}(\Gamma, \mathbb{C})$ with $L(\gamma)=0$ for all $\gamma$ in $\Gamma_{0}$. If

$$
\int_{\Gamma_{0} \backslash \mathbb{H}}\left(1+\left|\Lambda_{L}^{+}(z)\right|+\left|\Lambda_{L}^{-}(z)\right|\right)|\phi(z)| y^{k / 2} d \mu z<\infty
$$

then

$$
P[\phi, L](z):=\sum_{\gamma \in \Gamma_{0} \backslash \Gamma} L(\gamma)\left(\left.\phi\right|_{k} \gamma\right)(z)
$$

converges absolutely and uniformly on compact subsets of $\mathbb{H}$ to an element of $S_{k}^{2}$.

The functions $\Lambda_{L}^{+}(z)$ and $\Lambda_{L}^{-}(z)$ above satisfy

$$
L(\gamma)=\Lambda_{L}^{+}(\gamma z)-\Lambda_{L}^{+}(z)+\overline{\Lambda_{L}^{-}(\gamma z)-\Lambda_{L}^{-}(z)}
$$

for all $\gamma \in \Gamma$ and all $z \in \mathbb{C}$. See (8.4) for their definition.

Theorem 2 allows us to construct parabolic, hyperbolic and elliptic second-order Poincaré series. In Section 9 we show that, whenever they exist, these Poincaré series of order 1 and 2 always span their respective cusp form spaces. In the final section we speculate on the situation for third- and higher-order forms.

\section{Parabolic expansions}

2.1. All the properties of Fuchsian groups used in this paper are explained in [16], [25, Chapter 1] and [12, Chapter 2]. We say that $\gamma$ is a parabolic element of $\Gamma$ if its $\operatorname{trace}, \operatorname{tr}(\gamma)$, has absolute value 2. Then $\gamma$ fixes one point in $\mathbb{R} \cup\{\infty\}$. These parabolic fixed points are the cusps of $\Gamma$. If $\mathfrak{a}$ is a cusp of $\Gamma$ then the subgroup, $\Gamma_{\mathfrak{a}}$, of all elements in $\Gamma$ that fix $\mathfrak{a}$ is isomorphic to $\mathbb{Z}$. Thus $\Gamma_{\mathfrak{a}}=\left\langle\gamma_{\mathfrak{a}}\right\rangle$ for a parabolic generator $\gamma_{\mathfrak{a}} \in \Gamma$. There exists a scaling matrix $\sigma_{\mathfrak{a}} \in \mathrm{SL}_{2}(\mathbb{R})$ so that $\sigma_{\mathfrak{a}} \infty=\mathfrak{a}$ and

$$
\sigma_{\mathfrak{a}}^{-1} \Gamma_{\mathfrak{a}} \sigma_{\mathfrak{a}}=\left\{ \pm\left(\begin{array}{cc}
1 & m \\
0 & 1
\end{array}\right): m \in \mathbb{Z}\right\}
$$


The matrix $\sigma_{\mathfrak{a}}$ is unique up to multiplication on the right by any $\left(\begin{array}{ll}1 & x \\ 0 & 1\end{array}\right)$ with $x \in \mathbb{R}$. We label the group (2.1) as $\Gamma_{\infty}$. A natural fundamental domain for $\Gamma_{\infty} \backslash \mathbb{H}$ is the set $\mathbb{F}_{\infty}$ of all $z \in \mathbb{H}$ with $0 \leq \operatorname{Re}(z)<1$. The image of this set under $\sigma_{\mathfrak{a}}$ will be a fundamental domain for $\Gamma_{\mathfrak{a}} \backslash \mathbb{H}$ as shown in Figure 1.

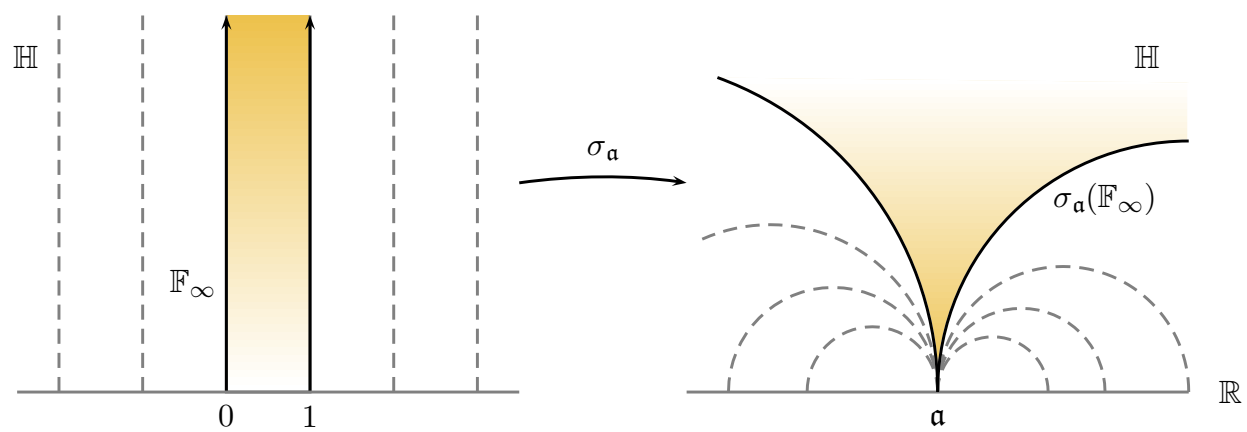

Fig. 1. The parabolic scaling map

We next define an operator $A$ that converts functions with a particular parabolic invariance into functions with invariance as $z \mapsto z+1$. Similar, though slightly more elaborate, operators will do the same for functions with hyperbolic and elliptic invariance in Sections 3, 4 .

Lemma 3. For any function $f$ with $\left.f\right|_{k} \gamma_{\mathfrak{a}}=f$, define

$$
A_{\mathfrak{a}} f:=\left(\left.f\right|_{k} \sigma_{\mathfrak{a}}\right) .
$$

Then $\left(A_{\mathfrak{a}} f\right)(z+1)=\left(A_{\mathfrak{a}} f\right)(z)$.

Proof. We have

$$
\begin{aligned}
\left(A_{\mathfrak{a}} f\right)(z+1) & =\left(\left.f\right|_{k} \sigma_{\mathfrak{a}}\right)\left(\left(\begin{array}{ll}
1 & 1 \\
0 & 1
\end{array}\right) z\right)=\left(\left.\left(\left.f\right|_{k} \sigma_{\mathfrak{a}}\left(\begin{array}{ll}
1 & 1 \\
0 & 1
\end{array}\right) \sigma_{\mathfrak{a}}^{-1}\right)\right|_{k} \sigma_{\mathfrak{a}}\right)(z) \\
& =\left(\left.\left(\left.f\right|_{k} \gamma_{\mathfrak{a}}\right)\right|_{k} \sigma_{\mathfrak{a}}\right)(z)=\left(A_{\mathfrak{a}} f\right)(z) .
\end{aligned}
$$

It follows that $f$ in $S_{k}$ or $S_{k}^{n}$ implies $\left(A_{\mathfrak{a}} f\right)(z)$ has period 1 and is holomorphic on $\mathbb{H}$. It consequently has a Fourier expansion

$$
\left(A_{\mathfrak{a}} f\right)(z)=\sum_{m \in \mathbb{Z}} b_{\mathfrak{a}}(m) e^{2 \pi i m z} .
$$

The rapid decay condition at the cusp $\mathfrak{a}$ in the definitions of $S_{k}$ and $S_{k}^{n}$ is then

$$
\left(A_{\mathfrak{a}} f\right)(z)=\left(\left.f\right|_{k} \sigma_{\mathfrak{a}}\right)(z) \ll_{\mathfrak{a}} e^{-c y}
$$

as $y \rightarrow \infty$ uniformly in $x$ for some constant $c>0$. This must hold at each of the cusps $\mathfrak{a}$. It is equivalent to $\left.f\right|_{k} \sigma_{\mathfrak{a}}$ only having terms with $m \geq 1$ in the expansion (2.2). We make the following definition, valid for all $f \in S_{k}^{n}$. 
Definition 2. For $f \in S_{k}^{n}$, the parabolic expansion of $f$ at $\mathfrak{a}$ is

$$
\left(\left.f\right|_{k} \sigma_{\mathfrak{a}}\right)(z)=\sum_{m \in \mathbb{N}} b_{\mathfrak{a}}(m) e^{2 \pi i m z}
$$

Suppose $\mathfrak{b}=\gamma \mathfrak{a}$ is another cusp $\Gamma$-equivalent to $\mathfrak{a}$. Then

$$
\Gamma_{\mathfrak{b}}=\Gamma_{\gamma \mathfrak{a}}=\gamma \Gamma_{\mathfrak{a}} \gamma^{-1}
$$

and $\sigma_{\mathfrak{b}}=\sigma_{\gamma \mathfrak{a}}=\gamma \sigma_{\mathfrak{a}}$. We have

$$
\begin{aligned}
\left(\left.f\right|_{k} \sigma_{\mathfrak{b}}\right)(z) & =j\left(\gamma \sigma_{\mathfrak{a}}, z\right)^{-k} f\left(\gamma \sigma_{\mathfrak{a}} z\right)=j\left(\sigma_{\mathfrak{a}}, z\right)^{-k}\left(\left.f\right|_{k} \gamma\right)\left(\sigma_{\mathfrak{a}} z\right) \\
& =j\left(\sigma_{\mathfrak{a}}, z\right)^{-k}\left(f\left(\sigma_{\mathfrak{a}} z\right)+f^{*}\left(\sigma_{\mathfrak{a}} z\right)\right) \\
& =\left(\left.f\right|_{k} \sigma_{\mathfrak{a}}\right)(z)+\left(\left.f^{*}\right|_{k} \sigma_{\mathfrak{a}}\right)(z)
\end{aligned}
$$

for $\left.f\right|_{k}(\gamma-1)=f^{*}$. Thus, if $f \in S_{k}$ then $f^{*}=0$ and its parabolic expansions at $\Gamma$-equivalent cusps are identical. If $f \in S_{k}^{n}$, its parabolic expansions at $\Gamma$-equivalent cusps are the same up to addition of the parabolic expansion of an element of $S_{k}^{n-1}$. Therefore, when testing whether $f \in S_{k}^{n}$, if $f$ satisfies condition (1.2) in the definition of $S_{k}^{n}$ then the rapid decay condition need only be verified at the finite number of $\Gamma$-inequivalent cusps of $\Gamma$.

2.2. It is easy to see that the reasoning in Lemma 3 may be reversed. If $g(z)$ has period 1 then

$$
A_{\mathfrak{a}}^{-1} g:=\left.g\right|_{k}\left(\sigma_{\mathfrak{a}}^{-1}\right)
$$

satisfies $\left.\left(A_{\mathfrak{a}}^{-1} g\right)\right|_{k} \gamma_{\mathfrak{a}}=A_{\mathfrak{a}}^{-1} g$. Therefore $\left.\left(A_{\mathfrak{a}}^{-1} g\right)\right|_{k} \gamma=A_{\mathfrak{a}}^{-1} g$ for all $\gamma \in \Gamma_{\mathfrak{a}}$. If we have a holomorphic function $g$ on $\mathbb{H}$ with period 1 then setting $\phi=A_{\mathfrak{a}}^{-1} g$ and $\Gamma_{0}=\Gamma_{\mathfrak{a}}$ in Theorem 1 gives us a natural candidate for a relative Poincaré series. The obvious periodic functions to use are the ones appearing on the right of (2.4): $e^{2 \pi i m z}$. So, recalling definition (1.5), we set

$$
\begin{aligned}
\Phi_{\operatorname{Par}}(z, m, \mathfrak{a}): & =P\left[A_{\mathfrak{a}}^{-1} e^{2 \pi i m \cdot}\right](z) \\
& =\sum_{\gamma \in \Gamma_{\mathfrak{a}} \backslash \Gamma}\left(\left.\left(A_{\mathfrak{a}}^{-1} e^{2 \pi i m \cdot}\right)\right|_{k} \gamma\right)(z)=\sum_{\gamma \in \Gamma_{\mathfrak{a}} \backslash \Gamma} \frac{e^{2 \pi i m\left(\sigma_{\mathfrak{a}}^{-1} \gamma z\right)}}{j\left(\sigma_{\mathfrak{a}}^{-1} \gamma, z\right)^{k}} .
\end{aligned}
$$

The next proposition is also proved in [12, Sections 3.1, 3.2], for example.

Proposition 4. For $4 \leq k \in 2 \mathbb{Z}$ and $m \in \mathbb{N}$ we have $\Phi_{\mathrm{Par}}(z, m, \mathfrak{a}) \in S_{k}$. 
Proof. With Theorem 1 and the discussion leading to (2.6) we need only confirm that (1.4) holds for $\Gamma_{0}=\Gamma_{\mathfrak{a}}$ and $\phi=A_{\mathfrak{a}}^{-1} e^{2 \pi i m}$. We have

$$
\begin{aligned}
\int_{\Gamma_{\mathfrak{a}} \backslash \mathbb{H}}|\phi(z)| y^{k / 2} d \mu z & =\int_{\Gamma_{\infty} \backslash \mathbb{H}}\left|\phi\left(\sigma_{\mathfrak{a}} z\right)\right| \operatorname{Im}\left(\sigma_{\mathfrak{a}} z\right)^{k / 2} d \mu z \\
& =\int_{0}^{\infty} \int_{0}^{1}\left|\frac{e^{2 \pi i m z}}{j\left(\sigma_{\mathfrak{a}}^{-1}, \sigma_{\mathfrak{a}} z\right)^{k}}\right| \frac{y^{k / 2}}{\left|j\left(\sigma_{\mathfrak{a}}, z\right)\right|^{k}} d \mu z \\
& =\int_{0}^{\infty} e^{-2 \pi m y} y^{k / 2-2} d y
\end{aligned}
$$

and (2.7) is bounded for $m \geq 1$ and $k>2$.

The vector space $S_{k}$ is equipped with a natural inner product, due to Petersson in [23],

$$
\left\langle f_{1}, f_{2}\right\rangle:=\int_{\Gamma \backslash \mathbb{H}} f_{1}(z) \overline{f_{2}(z)} y^{k} d \mu z .
$$

The following well-known result is demonstrated in [12, Section 3.3], for example, using the unfolding technique. We give the hyperbolic and elliptic versions in the next sections.

Proposition 5. For $4 \leq k \in 2 \mathbb{Z}, m \in \mathbb{N}$ and $f \in S_{k}$ satisfying (2.4),

$$
\left\langle f, \Phi_{\operatorname{Par}}(\cdot, m, \mathfrak{a})\right\rangle=b_{\mathfrak{a}}(m)\left[\frac{(k-2) !}{(4 \pi m)^{k-1}}\right] .
$$

\section{Hyperbolic expansions}

3.1. This material is based on Petersson's work in [24], see also [10]. An element $\gamma$ of $\Gamma$ is hyperbolic if $|\operatorname{tr}(\gamma)|>2$. Denote the set of all such elements by $\operatorname{Hyp}(\Gamma)$. Let $\eta=\left\{\eta_{1}, \eta_{2}\right\}$ be a hyperbolic pair of points in $\mathbb{R} \cup\{\infty\}$ for $\Gamma$. By this we mean that there exists one element of $\operatorname{Hyp}(\Gamma)$ that fixes each of $\eta_{1}, \eta_{2}$. The set of all such $\gamma$ is a group which we label $\Gamma_{\eta}$. As in the parabolic case this group is isomorphic to $\mathbb{Z}$ (see [16, Theorem 2.3.5] for the proof), and $\Gamma_{\eta}=\left\langle\gamma_{\eta}\right\rangle$. There exists a scaling matrix $\sigma_{\eta} \in \mathrm{SL}_{2}(\mathbb{R})$ such that $\sigma_{\eta} 0=\eta_{1}, \sigma_{\eta} \infty=\eta_{2}$ and

$$
\sigma_{\eta}^{-1} \gamma_{\eta} \sigma_{\eta}= \pm\left(\begin{array}{cc}
\xi & \\
& \xi^{-1}
\end{array}\right)
$$

for $\xi \in \mathbb{R}$. This scaling matrix $\sigma_{\eta}$ is unique up to multiplication on the right by any $\left(\begin{array}{cc}x & 0 \\ 0 & x^{-1}\end{array}\right)$ with $x \in \mathbb{R}$. Replacing the generator $\gamma_{\eta}$ by $\gamma_{\eta}^{-1}$ if necessary we may assume $\xi^{2}>1$. Let

$$
\mathbb{F}_{\eta}:=\left\{z \in \mathbb{H}: 1 \leq|z|<\xi^{2}\right\} .
$$


Then it is easy to see that $\sigma_{\eta}\left(\mathbb{F}_{\eta}\right)$ is a fundamental domain for $\Gamma_{\eta} \backslash \mathbb{H}$. See Figure 2.

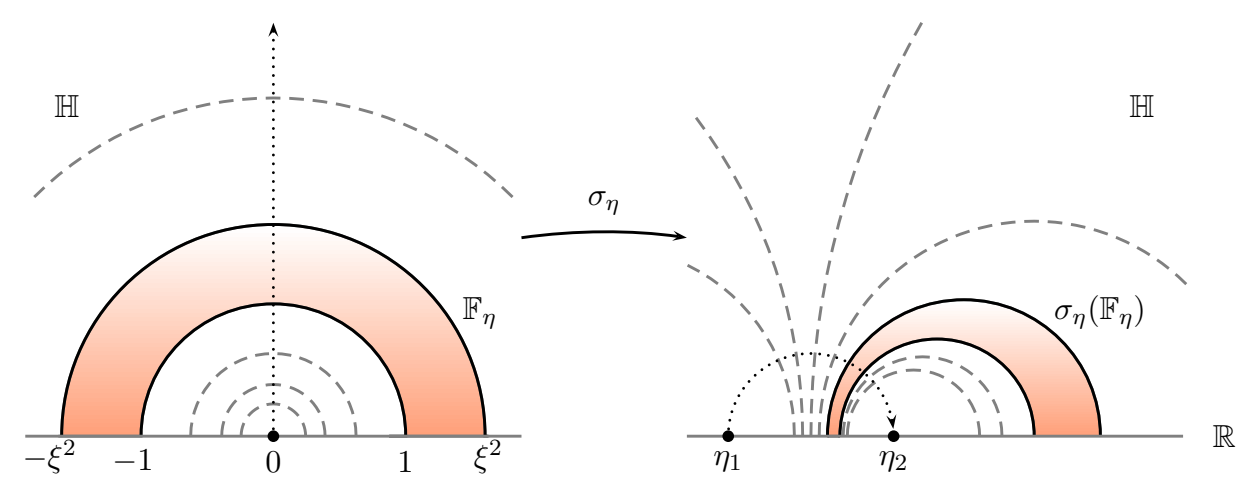

Fig. 2. The hyperbolic scaling map

Lemma 6. For any function $f$ with $\left.f\right|_{k} \gamma_{\eta}=f$, let

$$
\left(A_{\eta} f\right)(z):=\xi^{k z}\left(\left.f\right|_{k} \sigma_{\eta}\right)\left(\xi^{2 z}\right) .
$$

Then $\left(A_{\eta} f\right)(z+1)=\left(A_{\eta} f\right)(z)$.

Proof. First note that

$$
f\left(\left(\begin{array}{c}
\xi \\
\xi^{-1}
\end{array}\right) z\right)=\xi^{-k}\left(\left.f\right|_{k}\left({ }^{\xi}{ }_{\xi^{-1}}\right)\right)(z)
$$

and with a calculation,

$$
\begin{aligned}
\left(A_{\eta} f\right)(z+1) & =\xi^{k(z+1)}\left(\left.f\right|_{k} \sigma_{\eta}\right)\left(\xi^{2(z+1)}\right)=\xi^{k} \xi^{k z}\left(\left.f\right|_{k} \sigma_{\eta}\right)\left(\left(\begin{array}{c}
\xi \\
\xi^{-1}
\end{array}\right) \xi^{2 z}\right) \\
& =\xi^{k z}\left(\left.\left(\left.f\right|_{k} \sigma_{\eta}\left({ }^{\xi} \xi^{-1}\right) \sigma_{\eta}^{-1}\right)\right|_{k} \sigma_{\eta}\right)\left(\xi^{2 z}\right)=\xi^{k z}\left(\left.\left(\left.f\right|_{k} \gamma_{\eta}\right)\right|_{k} \sigma_{\eta}\right)\left(\xi^{2 z}\right) \\
& =\xi^{k z}\left(\left.f\right|_{k} \sigma_{\eta}\right)\left(\xi^{2 z}\right)=\left(A_{\eta} f\right)(z) .
\end{aligned}
$$

If $f \in S_{k}$ then $A_{\eta} f$ has period 1 and hence a Fourier expansion

$$
\left(A_{\eta} f\right)(z)=\sum_{m \in \mathbb{Z}} b_{\eta}(m) e^{2 \pi i m z}
$$

Put $w=\xi^{2 z}$ so that $e^{2 \pi i z}=w^{\pi i / \log \xi}$. Then (3.2) implies that $f \in S_{k}$ must have the following expansion:

DeFinition 3. The hyperbolic expansion of $f \in S_{k}$ at $\eta$ is

$$
\left(\left.f\right|_{k} \sigma_{\eta}\right)(w)=\sum_{m \in \mathbb{Z}} b_{\eta}(m) w^{-k / 2+\pi i m / \log \xi} .
$$

Petersson introduced this expansion in [24]. It is also valid for $f \in S_{k}^{n}$ provided $\left.f\right|_{k}(\gamma-1)=0$ for all $\gamma \in \Gamma_{\eta}$. 
3.2. Reversing Lemma 6 we see that if $g(z+1)=g(z)$ then the inverse operator to $A_{\eta}$ acts as follows:

$$
A_{\eta}^{-1} g:=\left.\left(B_{\eta} g\right)\right|_{k}\left(\sigma_{\eta}^{-1}\right) \quad \text { for }\left(B_{\eta} g\right)(z):=z^{-k / 2} g\left(\frac{\log z}{2 \log \xi}\right)
$$

and

$$
\left.\left(A_{\eta}^{-1} g\right)\right|_{k} \gamma_{\eta}=A_{\eta}^{-1} g
$$

Set

$$
\begin{aligned}
& \Phi_{\mathrm{Hyp}}(z, m, \eta):=P\left[A_{\eta}^{-1} e^{2 \pi i m \cdot}\right](z) \\
& =\sum_{\gamma \in \Gamma_{\eta} \backslash \Gamma}\left(\left.\left(A_{\eta}^{-1} e^{2 \pi i m \cdot}\right)\right|_{k} \gamma\right)(z)=\sum_{\gamma \in \Gamma_{\eta} \backslash \Gamma} \frac{\left(\sigma_{\eta}^{-1} \gamma z\right)^{-k / 2+\pi i m / \log \xi}}{j\left(\sigma_{\eta}^{-1} \gamma, z\right)^{k}} .
\end{aligned}
$$

Proposition 7. For $4 \leq k \in 2 \mathbb{Z}$ and $m \in \mathbb{Z}$ we have $\Phi_{\mathrm{Hyp}}(z, m, \eta) \in S_{k}$.

Proof. To apply Theorem 1 we must verify (1.4). We have

$$
\begin{aligned}
\int_{\Gamma_{\eta} \backslash \mathbb{H}}\left|\frac{\left(\sigma_{\eta}^{-1} z\right)^{-k / 2+\pi i m / \log \xi}}{j\left(\sigma_{\eta}^{-1}, z\right)^{k}}\right| y^{k / 2} d \mu z & =\int_{\mathbb{F}_{\eta}}\left|w^{-k / 2+\pi i m / \log \xi}\right| \operatorname{Im}(w)^{k / 2} d \mu w \\
& =\int_{1}^{\xi^{2}} \int_{0}^{\pi} r^{-k / 2} e^{-\pi m \theta / \log \xi}(r \sin \theta)^{k / 2-2} r d \theta d r \\
& =2 \log \xi \int_{0}^{\pi} e^{-\pi m \theta / \log \xi}(\sin \theta)^{k / 2-2} d \theta
\end{aligned}
$$

This is bounded for all $m \in \mathbb{Z}$ and $k>2$ as required.

Proposition 8. For $4 \leq k \in 2 \mathbb{Z}, m \in \mathbb{Z}$ and $f \in S_{k}$ satisfying (3.3),

$$
\left\langle f, \Phi_{\mathrm{Hyp}}(\cdot, m, \eta)\right\rangle=b_{\eta}(m)\left[2 \log \xi \int_{0}^{\pi} e^{-2 \pi m \theta / \log \xi}(\sin \theta)^{k-2} d \theta\right]
$$

$$
= \begin{cases}b_{\eta}(m) \cdot \frac{2 \log \xi}{(2 i)^{k-1}}\left(e^{-2 \pi^{2} m / \log \xi}-1\right) \frac{\Gamma\left(\frac{\pi i m}{\log \xi}-\frac{k}{2}+1\right) \Gamma(k-1)}{\Gamma\left(\frac{\pi m}{\log \xi}+\frac{k}{2}\right)} & \text { if } m \neq 0, \\
b_{\eta}(0) \cdot \frac{2 \pi \log \xi}{2^{k-2}}\left(\begin{array}{c}
k-2 \\
k / 2-1
\end{array}\right) & \text { if } m=0 .\end{cases}
$$


Proof. Unfold the inner product:

$$
\begin{aligned}
& \left\langle f, \Phi_{\mathrm{Hyp}}(\cdot, m, \eta)\right\rangle=\int_{\Gamma \backslash \mathbb{H}} y^{k} f(z) \overline{\Phi_{\mathrm{Hyp}}(z, m, \eta)} d \mu z \\
& =\int_{\Gamma \backslash \mathbb{H}} y^{k} f(z) \sum_{\gamma \in \Gamma_{\eta} \backslash \Gamma} \overline{\left(\left.\left(A_{\eta}^{-1} e^{2 \pi i m \cdot}\right)\right|_{k} \gamma\right)(z)} d \mu z \\
& =\int_{\Gamma \backslash \mathbb{H}} \sum_{\gamma \in \Gamma_{\eta} \backslash \Gamma} \operatorname{Im}(\gamma z)^{k} f(\gamma z) \overline{\left(A_{\eta}^{-1} e^{2 \pi i m \cdot}\right)(\gamma z)} d \mu z \\
& =\int_{\Gamma_{\eta} \backslash \mathbb{H}} f(z) \overline{\left(A_{\eta}^{-1} e^{2 \pi i m \cdot}\right)(z)} y^{k} d \mu z \\
& =\int_{\Gamma_{\eta} \backslash \mathbb{H}}\left(\frac{\sum_{l \in \mathbb{Z}} b_{\eta}(l)\left(\sigma_{\eta}^{-1} z\right)^{-k / 2+\pi i l / \log \xi}}{j\left(\sigma_{\eta}^{-1}, z\right)^{k}}\right) \frac{\overline{\left(\sigma_{\eta}^{-1} z\right)^{-k / 2+\pi i m / \log \xi}}}{\overline{j\left(\sigma_{\eta}^{-1}, z\right)}} y^{k} d \mu z \\
& =\sum_{l \in \mathbb{Z}} b_{\eta}(l) \int_{\Gamma_{\eta} \backslash \mathbb{H}}\left(\sigma_{\eta}^{-1} z\right)^{-k / 2+\pi i l / \log \xi} \overline{\left(\sigma_{\eta}^{-1} z\right)^{-k / 2+\pi i m / \log \xi}} \operatorname{Im}\left(\sigma_{\eta}^{-1} z\right)^{k} d \mu z .
\end{aligned}
$$

The integral is

$$
\begin{aligned}
\int_{\mathbb{F}_{\eta}} w^{-k / 2+\pi i l / \log \xi} \overline{w^{-k / 2+\pi i m / \log \xi}} \operatorname{Im}(w)^{k} d \mu w \\
=\int_{1}^{\xi^{2}} \int_{0}^{\pi}\left(r e^{i / \theta}\right)^{-k / 2+\pi i l / \log \xi} \overline{\left(r e^{i / \theta}\right)^{-k / 2+\pi i m / \log \xi}}(r \sin \theta)^{k-2} r d \theta d r \\
=\int_{0}^{\pi} e^{-\theta \pi(l+m) / \log \xi}(\sin \theta)^{k-2} d \theta \int_{1}^{\xi^{2}} r^{\pi i(l-m) / \log \xi} \frac{d r}{r}
\end{aligned}
$$

and letting $u=\log r / \log \xi$ we see that the last integral over $r$ is

$$
\log \xi \int_{0}^{2} e^{u \pi i(l-m)} d u= \begin{cases}2 \log \xi & \text { if } l=m \\ 0 & \text { if } l \neq m .\end{cases}
$$

Reassemble to complete the proof of (3.6). To evaluate (3.6) let

$$
\mathcal{I}_{a, b}:=\int_{0}^{\pi} e^{a \theta} \sin ^{b} \theta d \theta \quad \text { for } a \in \mathbb{C}, b \in 2 \mathbb{N}_{0} .
$$

For all $b \in 2 \mathbb{N}_{0}$ we have

$$
\mathcal{I}_{0, b}=\int_{0}^{\pi}\left(\frac{e^{i \theta}-e^{-i \theta}}{2 i}\right)^{b} d \theta=\frac{\pi}{2^{b}}\left(\begin{array}{c}
b \\
b / 2
\end{array}\right)
$$

using the binomial theorem. The $m=0$ case of (3.7) follows. For $a \neq 0$, we easily have $\mathcal{I}_{a, 0}=\left(e^{\pi a}-1\right) / a$. Using integration by parts twice we find, 
for $b \geq 2$,

Hence

$$
\frac{\mathcal{I}_{a, b}}{b !}=\frac{1}{a^{2}+b^{2}} \frac{\mathcal{I}_{a, b-2}}{(b-2) !} .
$$

$$
\begin{aligned}
\mathcal{I}_{a, b} & =\frac{\mathcal{I}_{a, 0} \cdot b !}{\left(a^{2}+b^{2}\right)\left(a^{2}+(b-2)^{2}\right) \cdots\left(a^{2}+2^{2}\right)} \\
& =\frac{\mathcal{I}_{a, 0} \cdot a \cdot b !}{(2 i)^{b+1}\left(\frac{a}{2 i}+\frac{b}{2}\right)\left(\frac{a}{2 i}+\frac{b}{2}-1\right) \cdots\left(\frac{a}{2 i}-\frac{b}{2}\right)} \\
& =\frac{\mathcal{I}_{a, 0} \cdot a \cdot \Gamma(b+1) \Gamma\left(\frac{a}{2 i}-\frac{b}{2}\right)}{(2 i)^{b+1} \Gamma\left(\frac{a}{2 i}+\frac{b}{2}+1\right)}
\end{aligned}
$$

and the $m \neq 0$ case of (3.7) is proved.

3.3. Various types of hyperbolic series have appeared in the literature. Associated to a hyperbolic element $\gamma_{\eta}=\left(\begin{array}{ll}a & b \\ c & d\end{array}\right)$ in $\Gamma$ we have the quadratic form

$$
Q_{\gamma_{\eta}}(z):=c z^{2}+(d-a) z-b
$$

of discriminant $D=(a+d)^{2}-4>0$ and with zeros $\eta=\left\{\eta_{1}, \eta_{2}\right\}$ at the fixed points of $\gamma_{\eta}$. We assume that $\gamma_{\eta}$ generates the subgroup of elements fixing $\eta$, that is, $\Gamma_{\eta}=\left\langle\gamma_{\eta}\right\rangle$. Also, since $Q_{\gamma_{\eta}}$ depends on the sign of the matrix entries, in this section we take $\Gamma \subset \mathrm{SL}_{2}(\mathbb{R})$ instead of $\mathrm{PSL}_{2}(\mathbb{R})$. Following Katok in $[15]$, we define the series

$$
\theta_{k, \gamma_{\eta}}(z):=\sum_{\gamma \in \Gamma_{\eta} \backslash \Gamma} \frac{1}{Q_{\gamma_{\eta}}(\gamma z)^{k / 2} j(\gamma, z)^{k}} .
$$

For $4 \leq k \in 2 \mathbb{Z}$ it is shown in [15] that $\theta_{k, \gamma_{\eta}} \in S_{k}$ and, moreover, that $S_{k}$ is spanned by $\theta_{k, \gamma}$ as $\gamma$ ranges over all hyperbolic elements of $\Gamma$. In [9] it is further shown that only those $\gamma$ that are words in the group generators of length at most $2^{k-1}-1$ are required for a spanning set.

Katok uses the series $\theta_{k, \gamma_{\eta}}$ to define a hyperbolic rational structure on $S_{k}$, analogous to the (parabolic) rational structure associated to the periods of cusp forms, as in [19]. For example, with $\Gamma=\mathrm{SL}_{2}(\mathbb{Z})$,

$$
C_{k, \gamma_{\eta}} \cdot\left\langle f, \theta_{k, \gamma_{\eta}}\right\rangle=r_{k}\left(f, \gamma_{\eta}\right)
$$

for any $f \in S_{k}$ where the right side is the hyperbolic period of $f$ associated to $\gamma_{\eta}$, defined as

$$
r_{k}\left(f, \gamma_{\eta}\right):=\int_{w}^{\gamma_{\eta} w} f(z) Q_{\gamma_{\eta}}(z)^{k / 2-1} d z
$$

and independent of $w \in \mathbb{H}$. On the left we have the normalization constant

$$
C_{k, \gamma_{\eta}}:=D^{(k-1) / 2} \frac{-\operatorname{sgn}\left(\operatorname{tr}\left(\gamma_{\eta}\right)\right)}{\pi}\left(\begin{array}{c}
k-2 \\
k / 2-1
\end{array}\right)^{-1} 2^{k / 2-2} .
$$

See [15] and [19] for further details. 
In [27], Zagier encounters the series

$$
F_{k, D}(z):=\sum_{b^{2}-4 a c=D} \frac{1}{\left(a z^{2}+b z+c\right)^{k / 2}}
$$

for $D \in \mathbb{N}$ where the sum is over all $(a, b, c) \in \mathbb{Z}^{3}$ with $\operatorname{gcd}(a, b, c)=1$. (We include the gcd condition for simplicity.) It is essentially the $D$ th Fourier coefficient of the holomorphic kernel function for the Shimura-Shintani correspondence between forms of integral and half-integral weight. We can express the quadratic form appearing in (3.9) as

$$
Q(z)=a z^{2}+b z+c=M_{Q}\left[\begin{array}{l}
z \\
1
\end{array}\right]:=\left(\begin{array}{l}
z \\
1
\end{array}\right)^{t} M_{Q}\left(\begin{array}{l}
z \\
1
\end{array}\right)
$$

for the matrix $M_{Q}:=\left(\begin{array}{cc}a & b / 2 \\ b / 2 & c\end{array}\right)$. We say two quadratic forms $Q, Q^{\prime}$ are equivalent if $M_{Q^{\prime}}=\gamma^{t} M_{Q} \gamma$ for some $\gamma \in \Gamma=\mathrm{SL}_{2}(\mathbb{Z})$. We denote by $[Q]$ the equivalence class of $Q$. The class number $h(D)<\infty$ is the number of equivalence classes of forms of discriminant $D$. For a quadratic form $Q$ of discriminant $D>0$ put

$$
F_{k, D,[Q]}(z)=\sum_{Q^{\prime} \in[Q]} \frac{1}{Q^{\prime}(z)^{k / 2}} .
$$

Thus we may break up $F_{k, D}$ into $h(D)$ pieces $F_{k, D,[Q]}$.

Proposition 9. For $\Gamma=\mathrm{SL}_{2}(\mathbb{Z})$ we have $F_{k, D,\left[Q_{\gamma_{\eta}}\right]}=\theta_{k, \gamma_{\eta}}$.

Proof. A short computation shows

$$
Q_{\gamma_{\eta}}(z)=\left(\left(\begin{array}{cc}
0 & 1 \\
-1 & 0
\end{array}\right) \gamma_{\eta}\right)\left[\begin{array}{l}
z \\
1
\end{array}\right]
$$

Therefore

$$
\left[Q_{\gamma_{\eta}}\right]=\bigcup_{\gamma \in \Gamma}\left(\gamma^{t}\left(\left(\begin{array}{cc}
0 & 1 \\
-1 & 0
\end{array}\right) \gamma_{\eta}\right) \gamma\right)\left[\begin{array}{l}
z \\
1
\end{array}\right] .
$$

To eliminate the repetition in the union, put

$$
\begin{aligned}
E & :=\left\{\gamma \in \Gamma: \gamma^{t}\left(\begin{array}{cc}
0 & 1 \\
-1 & 0
\end{array}\right) \gamma_{\eta} \gamma=\left(\begin{array}{cc}
0 & 1 \\
-1 & 0
\end{array}\right) \gamma_{\eta}\right\} \\
& =\left\{\gamma \in \Gamma: \gamma^{-1} \gamma_{\eta} \gamma=\gamma_{\eta}\right\} .
\end{aligned}
$$


Check that $E$ is a subgroup of $\Gamma$ and use (3.1) to show that $E=\Gamma_{\eta}$. Hence

$$
\begin{aligned}
F_{k, D,\left[Q_{\left.\gamma_{\eta}\right]}\right]}(z) & =\sum_{\gamma \in \Gamma_{\eta} \backslash \Gamma}\left(\left(\gamma^{t}\left(\left(\begin{array}{cc}
0 & 1 \\
-1 & 0
\end{array}\right) \gamma_{\eta}\right) \gamma\right)\left[\begin{array}{l}
z \\
1
\end{array}\right]\right)^{-k / 2} \\
& =\sum_{\gamma \in \Gamma_{\eta} \backslash \Gamma}\left(\left(\left(\begin{array}{cc}
0 & 1 \\
-1 & 0
\end{array}\right) \gamma_{\eta}\right)\left[\gamma\left(\begin{array}{l}
z \\
1
\end{array}\right)\right]\right)^{-k / 2} \\
& =\sum_{\gamma \in \Gamma_{\eta} \backslash \Gamma}\left(Q_{\gamma_{\eta}}(\gamma z) j(\gamma, z)^{2}\right)^{-k / 2} .
\end{aligned}
$$

Now we recognize that the series (3.8), (3.9), (3.10) of Katok and Zagier are, up to normalization, the Petersson hyperbolic Poincaré series with $m=0$ :

Proposition 10. We have $\theta_{k, \gamma_{\eta}}(z)=\left(\xi-\xi^{-1}\right)^{-k / 2} \Phi_{\mathrm{Hyp}}(z, 0, \eta)$.

Proof. With (3.1) we find

$$
Q_{\gamma_{\eta}}(z)=\left(\begin{array}{cc}
0 & -\xi^{-1} \\
\xi & 0
\end{array}\right)\left[\sigma_{\eta}^{-1}\left(\begin{array}{l}
z \\
1
\end{array}\right)\right]=\left(\xi-\xi^{-1}\right) j\left(\sigma_{\eta}^{-1}, z\right)^{2} \sigma_{\eta}^{-1} z .
$$

Therefore

$$
Q_{\gamma_{\eta}}(\gamma z)=\left(\xi-\xi^{-1}\right) \frac{j\left(\sigma_{\eta}^{-1} \gamma, z\right)^{2}}{j(\gamma, z)^{2}} \sigma_{\eta}^{-1} \gamma z
$$

and putting this into (3.8) and comparing with (3.5) finishes the proof.

To end this section, we briefly note that Siegel in [26, Chapter II, §3] found that the hyperbolic expansion coefficients of the non-holomorphic parabolic Eisenstein series are essentially Hecke Grössencharakter $L$-functions associated to a real quadratic field. For second-order non-holomorphic Eisenstein series the same computation is carried out in [2], leading to Hecke $L$-functions twisted by modular symbols.

\section{Elliptic expansions}

4.1. If $z_{0}=\alpha+i \beta$ in $\mathbb{H}$ is fixed by a non-identity element of $\Gamma$, it is called an elliptic point of $\Gamma$. Such group elements necessarily have traces with absolute value less than 2 (and are called elliptic elements). Let $\Gamma_{z_{0}} \subset \Gamma$ be the subgroup of all elements fixing $z_{0}$. As shown in [16, Theorem 2.3.5, Corollary 2.4.2] it is a cyclic group of finite order $N>1$. Let $\varepsilon \in \Gamma$ be a generator of $\Gamma_{z_{0}}$. There exists $\sigma_{z_{0}} \in \mathrm{GL}(2, \mathbb{C})$ so that $\sigma_{z_{0}} 0=z_{0}, \sigma_{z_{0}} \infty=\overline{z_{0}}$. To be explicit, we take

$$
\sigma_{z_{0}}=\frac{1}{2 i \beta}\left(\begin{array}{cc}
-\overline{z_{0}} & z_{0} \\
-1 & 1
\end{array}\right), \quad \sigma_{z_{0}}^{-1}=\left(\begin{array}{cc}
1 & -z_{0} \\
1 & -\overline{z_{0}}
\end{array}\right)
$$


Note that $\sigma_{z_{0}}^{-1}$ maps the upper half-plane $\mathbb{H}$ homeomorphically to the open unit disc $\mathbb{D}_{1} \subset \mathbb{C}$ centered at the origin. For any $w \in \mathbb{H}$ a calculation shows $\left(\sigma_{z_{0}}^{-1} \varepsilon \sigma_{z_{0}}\right) w=\zeta^{2} w$ with $\zeta=j\left(\varepsilon, \overline{z_{0}}\right)$ and

$$
\sigma_{z_{0}}^{-1} \varepsilon \sigma_{z_{0}}=\left(\begin{array}{cc}
\zeta & \\
& \zeta^{-1}
\end{array}\right)
$$

Hence $\zeta$ is a primitive $2 N$ th root of unity: $\zeta=e^{2 \pi i m /(2 N)}$ for some $m$ with $(m, 2 N)=1$. There exists $m^{\prime} \in \mathbb{N}$ so that $m^{\prime} m \equiv 1 \bmod 2 N$ and $\zeta^{m^{\prime}}=e^{\pi i / N}$. So, replacing $\varepsilon$ by $\varepsilon^{m^{\prime}}$ if necessary, we may assume $\zeta=e^{\pi i / N}$. Let $\mathbb{F}_{z_{0}}$ equal the central sector covering $1 / N$ th of the disc and chosen with angle $\theta$ satisfying $-\pi / N \leq \theta-\pi \leq \pi / N$, for example, as in Figure 3. Then $\sigma_{z_{0}}\left(\mathbb{F}_{z_{0}}\right)$ is a convenient fundamental domain for $\Gamma_{z_{0}} \backslash \mathbb{H}$. Also note that there exists $C\left(z_{0}, \Gamma\right)>0$ such that

$$
|z|<C\left(z_{0}, \Gamma\right) \quad \text { for all } z \in \sigma_{z_{0}}\left(\mathbb{F}_{z_{0}}\right) .
$$

In other words, the fundamental domain we have chosen is contained in a bounded region of $\mathbb{H}$. We will need this in the proof of Theorem 20.
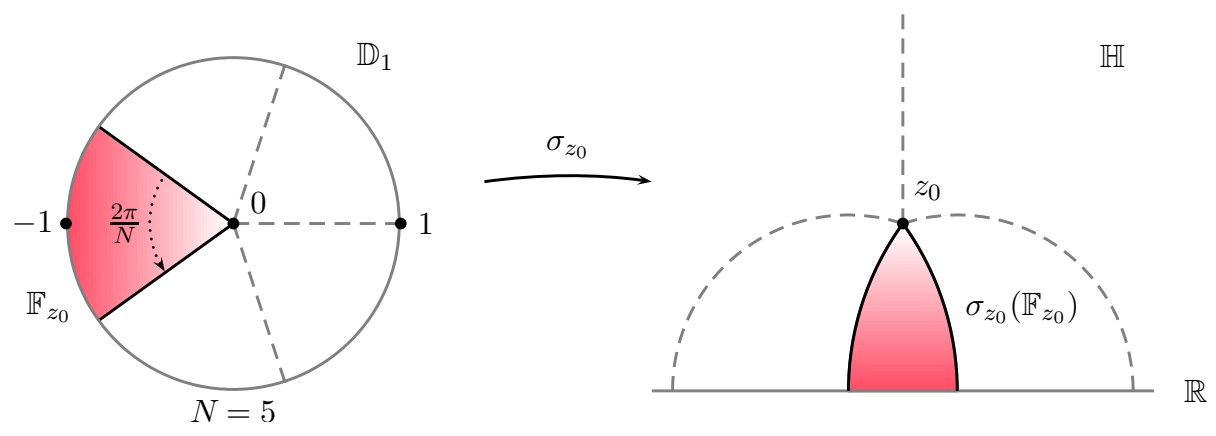

Fig. 3. The elliptic scaling map

Since any $f \in S_{k}(\Gamma)$ is holomorphic at $z=z_{0}$ we see that $f\left(\sigma_{z_{0}} w\right)$ is holomorphic at $w=0$ and has a Taylor series $\sum_{n} a_{z_{0}}(n) w^{n}$. Therefore we get the simple expansion

$$
f(z)=\sum_{n \in \mathbb{N}_{0}} a_{z_{0}}(n)\left(\sigma_{z_{0}}^{-1} z\right)^{n} .
$$

More useful for our purposes is the slightly different elliptic expansion due to Petersson. For $f, g: \mathbb{H} \rightarrow \mathbb{C}$ define

$$
\begin{aligned}
\left(A_{z_{0}} f\right)(z) & :=\zeta^{k z}\left(\left.f\right|_{k} \sigma_{z_{0}}\right)\left(\zeta^{2 z}\right), \\
A_{z_{0}}^{-1} g & :=\left.\left(B_{z_{0}} g\right)\right|_{k}\left(\sigma_{z_{0}}^{-1}\right), \quad\left(B_{z_{0}} g\right)(z):=z^{-k / 2} g\left(\frac{N \log z}{2 \pi i}\right) .
\end{aligned}
$$


A calculation identical to that of Lemma 6 and its reverse proves the following.

Lemma 11. We have $A_{z_{0}} A_{z_{0}}^{-1} f=A_{z_{0}}^{-1} A_{z_{0}} f=f$ and

$$
\begin{aligned}
\left(\left.f\right|_{k} \varepsilon\right)(z) & =f(z) \Rightarrow\left(A_{z_{0}} f\right)(z+1)=\left(A_{z_{0}} f\right)(z), \\
g(z+1) & =\left.g(z) \Rightarrow\left(A_{z_{0}}^{-1} g\right)\right|_{k} \varepsilon=A_{z_{0}}^{-1} g .
\end{aligned}
$$

Note that the matrices $\sigma_{z_{0}}$ and $\sigma_{z_{0}}^{-1}$ have determinants $1 /(2 i \beta)$ and $2 i \beta$ respectively. In this case it is convenient to normalize the stroke operator | and define

$$
\left(\left.f\right|_{k} \gamma\right)(z):=\frac{\operatorname{det}(\gamma)^{k / 2} f(\gamma z)}{j(\gamma, z)^{k}}
$$

Obviously, this agrees with our previous definition when $\gamma \in \mathrm{SL}_{2}(\mathbb{R})$.

Let $f \in S_{k}$; then $A_{z_{0}} f$ has period 1 and a Fourier expansion

$$
\left(A_{z_{0}} f\right)(z)=\sum_{m \in \mathbb{Z}} b_{z_{0}}(m) e^{2 \pi i m z} .
$$

Put $w=\zeta^{2 z}=e^{2 \pi i z / N}$ so that $e^{2 \pi i z}=w^{N}$ and

$$
\left(\left.f\right|_{k} \sigma_{z_{0}}\right)(w)=\sum_{m \in \mathbb{Z}} b_{z_{0}}(m) w^{N m-k / 2} .
$$

Since $\left(\left.f\right|_{k} \sigma_{z_{0}}\right)(w)$ is holomorphic at $w=0$, we must have non-negative powers of $w$ in the above expansion. Thus any $f \in S_{k}$ satisfies the following.

Definition 4. The elliptic expansion of $f$ in $S_{k}$ at $z_{0}$ is

$$
\left(\left.f\right|_{k} \sigma_{z_{0}}\right)(z)=\sum_{\substack{m \in \mathbb{N} \\ N m-k / 2 \geq 0}} b_{z_{0}}(m) z^{N m-k / 2} .
$$

We show in Lemma 17 that $\left.f\right|_{k} \varepsilon=f$ for all $f$ in $S_{k}^{n}$ also. Thus higherorder cusp forms also have the elliptic expansion (4.4). In some situations the exponent $N m-k / 2$ is a little awkward and we write

$$
\left(\left.f\right|_{k} \sigma_{z_{0}}\right)(z)=\sum_{l \in \mathbb{N}_{0}} c_{z_{0}}(l) z^{l}
$$

instead, where

$$
c_{z_{0}}(l)= \begin{cases}b_{z_{0}}\left(\frac{l+k / 2}{N}\right) & \text { if } l \equiv-k / 2 \bmod N \\ 0 & \text { if } l \not \equiv-k / 2 \bmod N\end{cases}
$$


4.2. Next define the elliptic Poincaré series

$$
\begin{aligned}
& \Phi_{\mathrm{Ell}}\left(z, m, z_{0}\right):=P\left[A_{z_{0}}^{-1} e^{2 \pi i m \cdot}\right](z)=\sum_{\gamma \in \Gamma_{z_{0}} \backslash \Gamma}\left(\left.\left(A_{z_{0}}^{-1} e^{2 \pi i m \cdot}\right)\right|_{k} \gamma\right)(z) \\
&=(2 i \beta)^{k / 2} \sum_{\gamma \in \Gamma_{z_{0}} \backslash \Gamma} \frac{\left(\sigma_{z_{0}}^{-1} \gamma z\right)^{N m-k / 2}}{j\left(\sigma_{z_{0}}^{-1} \gamma, z\right)^{k}} .
\end{aligned}
$$

Proposition 12. For $4 \leq k \in 2 \mathbb{Z}$ and $k /(2 N) \leq m \in \mathbb{N}$ we have $\Phi_{\mathrm{Ell}}\left(z, m, z_{0}\right) \in S_{k}$.

Proof. Recalling Theorem 1 we need to verify that

$$
\int_{\Gamma_{z_{0} \backslash \mathbb{H}}}|\phi(z)| y^{k / 2} d \mu z=(2 \beta)^{k / 2} \int_{\Gamma_{z_{0}} \backslash \mathbb{H}}\left|\frac{\left(\sigma_{z_{0}}^{-1} z\right)^{N m-k / 2}}{j\left(\sigma_{z_{0}}^{-1}, z\right)^{k}}\right| y^{k / 2} d \mu z
$$

is bounded. Put $w=u+i v=\sigma_{z_{0}}^{-1} z$ and recall that $z_{0}=\alpha+i \beta, z=x+i y$. We calculate

$$
\begin{aligned}
\left|z-\bar{z}_{0}\right|^{-2} & =\left|j\left(\sigma_{z_{0}}^{-1}, \sigma_{z_{0}} w\right)\right|^{-2}=\left|j\left(\sigma_{z_{0}}, w\right)\right|^{2}=(2 \beta)^{-2}|1-w|^{2}, \\
y & =\beta \frac{1-|w|^{2}}{|1-w|^{2}}, \quad\left|\frac{\partial(x, y)}{\partial(u, v)}\right|=\frac{4 \beta^{2}}{|1-w|^{4}}
\end{aligned}
$$

and see that (4.8) equals

$$
\begin{aligned}
2^{2-k / 2} \int_{\mathbb{F}_{z_{0}}}|w|^{N m-k / 2}\left(1-|w|^{2}\right)^{k / 2-2} d u d v & \\
& =2^{2-k / 2} \int_{0}^{1} \int_{\pi-\pi / N}^{\pi+\pi / N} r^{N m-k / 2+1}\left(1-r^{2}\right)^{k / 2-2} d \theta d r .
\end{aligned}
$$

This is bounded for $k>2$ and, by Theorem $1, \Phi_{\mathrm{Ell}}\left(z, m, z_{0}\right) \in S_{k}$.

As in the parabolic and hyperbolic cases, the elliptic Poincaré series can be used to determine the elliptic expansion coefficients. We follow closely the reasoning in [24] and first prove the following.

Lemma 13. For any integers $a, b \geq 0$ and $k \geq 2$,

$$
\int_{\mathbb{H}} \frac{\left(\sigma_{z_{0}}^{-1} z\right)^{a}\left(\overline{\sigma_{z_{0}}^{-1} z}\right)^{b}}{\left|j\left(\sigma_{z_{0}}^{-1}, z\right)\right|^{2 k}} y^{k} d \mu z= \begin{cases}\frac{4 \pi(k-2) ! a !}{(4 \beta)^{k}(a+k-1) !} & \text { if } a=b, \\ 0 & \text { if } a \neq b .\end{cases}
$$

Proof. With $w=\sigma_{z_{0}}^{-1} z$, the integral in (4.10) becomes

$$
\int_{\mathbb{D}_{1}} \frac{w^{a} \bar{w}^{b}}{\left|j\left(\sigma_{z_{0}}^{-1}, \sigma_{z_{0}} w\right)\right|^{2 k}} y^{k-2}\left|\frac{\partial(x, y)}{\partial(u, v)}\right| d u d v
$$


As in the proof of Proposition 12 this reduces to

$$
\frac{4}{(4 \beta)^{k}} \int_{\mathbb{D}_{1}} w^{a} \bar{w}^{b}\left(1-|w|^{2}\right)^{k-2} d u d v .
$$

Let $w=r e^{i \theta}$ and (4.11) becomes

$$
\frac{4}{(4 \beta)^{k}} \int_{0}^{1} \int_{0}^{2 \pi} r^{a+b+1}\left(1-r^{2}\right)^{k-2} e^{i \theta(a-b)} d \theta d r .
$$

Doing the integral over $\theta$ leaves a beta integral.

A similar proof (integrating over $\mathbb{F}_{z_{0}}$ instead of $\mathbb{D}_{1}$ ) shows

Lemma 14. For any integers $N l-k / 2, N m-k / 2 \geq 0$ and $k \geq 2$,

$$
\begin{aligned}
\int_{\Gamma_{z_{0}} \backslash \mathbb{H}} \frac{\left(\sigma_{z_{0}}^{-1} z\right)^{N l-k / 2}\left(\overline{\sigma_{z_{0}}^{-1} z}\right)^{N m-k / 2}}{\left|j\left(\sigma_{z_{0}}^{-1}, z\right)\right|^{2 k}} y^{k} d \mu z \\
= \begin{cases}\frac{4 \pi(k-2) !(N m-k / 2) !}{N(4 \beta)^{k}(N m+k / 2-1) !} & \text { if } l=m, \\
0 & \text { if } l \neq m .\end{cases}
\end{aligned}
$$

Proposition 15. For $f \in S_{k}$ satisfying (4.4) and $N m-k / 2 \geq 0$ we have

$$
\left\langle f, \Phi_{\mathrm{Ell}}\left(\cdot, m, z_{0}\right)\right\rangle=b_{z_{0}}(m)\left[\frac{\pi(k-2) !(N m-k / 2) !}{2^{k-2} N(N m+k / 2-1) !}\right] .
$$

Proof. Unfold the inner product as in Proposition 8:

$$
\begin{aligned}
& \left\langle f, \Phi_{\mathrm{Ell}}\left(\cdot, m, z_{0}\right)\right\rangle=\int_{\Gamma \backslash \mathbb{H}} y^{k} f(z) \overline{\Phi_{\mathrm{Ell}}\left(z, m, z_{0}\right)} d \mu z \\
& =\int_{\Gamma_{z_{0} \backslash \mathbb{H}}} f(z) \overline{\left(A_{z_{0}}^{-1} e^{2 \pi i m \cdot}\right)(z)} y^{k} d \mu z \\
& =(2 \beta)^{k} \int_{\Gamma_{z_{0}} \backslash \mathbb{H}}\left(\frac{\sum_{l \in \mathbb{Z}} b_{z_{0}}(l)\left(\sigma_{z_{0}}^{-1} z\right)^{N l-k / 2}}{j\left(\sigma_{z_{0}}^{-1}, z\right)^{k}}\right) \frac{\left(\overline{\sigma_{z_{0}}^{-1} z}\right)^{N m-k / 2}}{j\left(\sigma_{z_{0}}^{-1}, z\right)} y^{k} d \mu z \\
& =(2 \beta)^{k} \sum_{l \in \mathbb{Z}} b_{z_{0}}(l) \int_{\Gamma_{z_{0}} \backslash \mathbb{H}} \frac{\left(\sigma_{z_{0}}^{-1} z\right)^{N l-k / 2}\left(\overline{\sigma_{z_{0}}^{-1} z}\right)^{N m-k / 2}}{\left|j\left(\sigma_{z_{0}}^{-1}, z\right)\right|^{2 k}} y^{k} d \mu z \\
& =b_{z_{0}}(m)\left[\frac{\pi(k-2) !(N m-k / 2) !}{2^{k-2} N(N m+k / 2-1) !}\right] \text {. }
\end{aligned}
$$


4.3. Petersson in [24] defined the elliptic Poincaré series slightly differently as, essentially,

$$
\Phi_{\mathrm{Ell}}^{*}\left(z, l, z_{0}\right):=(2 i \beta)^{k / 2} \sum_{\gamma \in \Gamma} \frac{\left(\sigma_{z_{0}}^{-1} \gamma z\right)^{l}}{j\left(\sigma_{z_{0}}^{-1} \gamma, z\right)^{k}}
$$

for $l \in \mathbb{N}_{0}$. Notice that the sum is now over all elements of $\Gamma$. With the results of Section 4.2 and recalling (4.5) we see that

$$
\left\langle f, \Phi_{\mathrm{Ell}}^{*}\left(\cdot, l, z_{0}\right)\right\rangle=c_{z_{0}}(l)\left[\frac{\pi(k-2) ! m !}{2^{k-2}(m+k-1) !}\right] .
$$

Thus (4.6) implies that $\Phi_{\mathrm{Ell}}^{*}\left(\cdot, l, z_{0}\right)$ is orthogonal to all of $S_{k}$, and hence zero, unless $l \equiv-k / 2 \bmod N$. For such $l$,

$$
\begin{aligned}
\Phi_{\mathrm{Ell}}^{*}\left(z, l, z_{0}\right) & =(2 i \beta)^{k / 2} \sum_{\gamma \in \Gamma \backslash \Gamma_{z_{0}}} \sum_{\varepsilon \in \Gamma_{z_{0}}} \frac{\left(\sigma_{z_{0}}^{-1} \gamma \varepsilon z\right)^{l}}{j\left(\sigma_{z_{0}}^{-1} \gamma \varepsilon, z\right)^{k}} \\
& =\sum_{i=0}^{N-1}\left(\left.\Phi_{\mathrm{Ell}}\left(\cdot, l, z_{0}\right)\right|_{k} \varepsilon^{i}\right)(z)=N \Phi_{\mathrm{Ell}}\left(z, l, z_{0}\right) .
\end{aligned}
$$

For $l \in \mathbb{N}_{0}$ we have shown

$$
\Phi_{\mathrm{Ell}}^{*}\left(z, l, z_{0}\right)= \begin{cases}N \Phi_{\mathrm{Ell}}\left(z,(l+k / 2) / N, z_{0}\right) & \text { if } l \equiv-k / 2 \bmod N \\ 0 & \text { if } l \not \equiv-k / 2 \bmod N .\end{cases}
$$

5. Relative Poincaré series. We now give the proof of Theorem 1, of the relative Poincaré series construction.

Proof of Theorem 1. Define the open hyperbolic ball of center $z_{0}$ and radius $r$ as

$$
\mathbb{B}\left(z_{0}, r\right):=\left\{z \in \mathbb{H}: \rho\left(z, z_{0}\right)<r\right\}
$$

with hyperbolic distance $\rho\left(z, z_{0}\right)=\log \left(\left(1+\left|\sigma_{z_{0}}^{-1} z\right|\right) /\left(1-\left|\sigma_{z_{0}}^{-1} z\right|\right)\right)$. Then $\sigma_{z_{0}}^{-1} \mathbb{B}\left(z_{0}, r\right)=\mathbb{D}_{R}$ for

$$
R=\frac{e^{r}-1}{e^{r}+1}<1, \quad \mathbb{D}_{R}=\{z \in \mathbb{C}:|z|<R\} .
$$

A proof very similar to that of Lemma 13 shows

$$
\int_{\mathbb{B}\left(z_{0}, r\right)}\left(\sigma_{z_{0}}^{-1} z\right)^{n} \frac{y^{k / 2}}{\left|z-\overline{z_{0}}\right|^{k}} d \mu z= \begin{cases}C_{r, k} \beta^{-k / 2} & \text { if } n=0, \\ 0 & \text { if } n \in \mathbb{N},\end{cases}
$$

for $k \geq 2, \beta=\operatorname{Im}\left(z_{0}\right)$ and

$$
C_{r, k}=\pi 2^{k-3}\left(1-\left(1-R^{2}\right)^{k / 2-1}\right) /(k-2)
$$


for $k>2$. When $k=2, C_{r, k}=-\pi \log \left(1-R^{2}\right)$. Recall the expansion (4.2) and note that $a_{z_{0}}(0)=f\left(z_{0}\right)$. With (5.1) we find

$$
a_{z_{0}}(0) C_{r, k} \beta^{-k / 2}=\int_{\mathbb{B}\left(z_{0}, r\right)} f(z) \frac{y^{k / 2}}{\left|z-\overline{z_{0}}\right|^{k}} d \mu z
$$

giving a type of hyperbolic holomorphic mean-value result (replace $z$ by $w$ and $z_{0}$ by $z$ ):

$$
y^{-k / 2} f(z)=\frac{1}{C_{r, k}} \int_{\mathbb{B}(z, r)} f(w) \frac{\operatorname{Im}(w)^{k / 2}}{|w-\bar{z}|^{k}} d \mu w
$$

valid for any $f$ holomorphic on $\mathbb{B}(z, r)$ with real $k \geq 2$. Since $\operatorname{Im}(w)>0$ and $\operatorname{Im}(\bar{z})=-y$, we have $|w-\bar{z}|>y$ and therefore

$$
y^{k / 2}|f(z)| \leq \frac{1}{C_{r, k}} \int_{\mathbb{B}(z, r)}|f(w)| \operatorname{Im}(w)^{k / 2} d \mu w .
$$

We have $\gamma \mathbb{B}\left(z_{0}, r\right)=\mathbb{B}\left(\gamma z_{0}, r\right)$ for all $\gamma$ in $\Gamma$. As shown in [25, Proposition 1.8], there exists an $r>0$ so that $\mathbb{B}\left(\gamma z_{0}, r\right) \cap \mathbb{B}\left(\gamma^{\prime} z_{0}, r\right)=\emptyset$ for all $\gamma \neq \gamma^{\prime} \in$ $\Gamma_{0} \backslash \Gamma$. With this choice of $r$ we see that

$$
\begin{aligned}
y^{k / 2}|P[\phi](z)| & =\left|y^{k / 2} \sum_{\gamma \in \Gamma_{0} \backslash \Gamma}\left(\left.\phi\right|_{k} \gamma\right)(z)\right| \leq \sum_{\gamma \in \Gamma_{0} \backslash \Gamma} \operatorname{Im}(\gamma z)^{k / 2}|\phi(\gamma z)| \\
& \leq \sum_{\gamma \in \Gamma_{0} \backslash \Gamma} \frac{1}{C_{r, k}} \int_{\mathbb{B}(\gamma z, r)}|\phi(w)| \operatorname{Im}(w)^{k / 2} d \mu w \\
& \leq \frac{1}{C_{r, k}} \int_{\Gamma_{0} \backslash \mathbb{H}}|\phi(w)| \operatorname{Im}(w)^{k / 2} d \mu w<\infty,
\end{aligned}
$$

so $P[\phi]$ converges absolutely and uniformly to a holomorphic function on $\mathbb{H}$. At this point we may verify that $\left.P[\phi]\right|_{k}(\gamma-1)=0$ for all $\gamma \in \Gamma$. Also we have shown that $y^{k / 2}|P[\phi](z)|$ is bounded and it follows from this (see [12, p. 70]) for example, that $P[\phi]$ has rapid decay at each cusp. Hence $P[\phi] \in S_{k}$ as we wanted to show.

6. An elliptic expansion example. For $q=e^{2 \pi i z}$, the discriminant function is

$$
\Delta(z):=q \prod_{n=1}^{\infty}\left(1-q^{n}\right)^{24} .
$$

It generates the one-dimensional space $S_{12}\left(\Gamma_{0}(1)\right)$ for $\Gamma_{0}(1)=\mathrm{PSL}_{2}(\mathbb{Z})$. Its parabolic expansion at infinity is

$$
\Delta(z)=\sum_{m=1}^{\infty} \tau(m) q^{m}
$$


and Ramanujan discovered that its coefficients $\tau(m) \in \mathbb{Z}$ have many remarkable properties. The point $z_{0}=i \in \mathbb{H}$ is an elliptic point for $\Gamma_{0}(1)$. It is fixed by $\varepsilon= \pm\left(\begin{array}{cc}0 & -1 \\ 1 & 0\end{array}\right)$ with order $N=2$. Then, recalling (4.5), the elliptic expansion of $\Delta$ at $i$ is

$$
\left(\left.\Delta\right|_{12} \sigma_{i}\right)(w)=\sum_{m=0}^{\infty} c_{i}(m) w^{m} .
$$

Do the coefficients $c_{i}(m)$ have any arithmetic properties? As Petersson realized in [24], it is possible to prove a general result relating the elliptic expansion coefficients at a point to the Taylor coefficients there.

Proposition 16. For $f \in S_{k}$ with elliptic expansion (4.5) at $z_{0} \in \mathbb{H}$,

$$
c_{z_{0}}(m)=\sum_{r=0}^{m}\left(\begin{array}{c}
m+k-1 \\
r+k-1
\end{array}\right) \frac{\left(z_{0}-\overline{z_{0}}\right)^{r+k / 2}}{r !} f^{(r)}\left(z_{0}\right) .
$$

Proof. Since $f(z)$ is holomorphic in a neighborhood of $z_{0}$ it has a Taylor expansion

$$
f(z)=f\left(z_{0}\right)+\left(z-z_{0}\right) f^{\prime}\left(z_{0}\right)+\left(z-z_{0}\right)^{2} f^{\prime \prime}\left(z_{0}\right) / 2 !+\cdots .
$$

With (4.5) we have

$$
\left(\left.f\right|_{k} \sigma_{z_{0}}\right)(w)=\frac{(2 i \beta)^{k / 2}}{(1-w)^{k}} f\left(\frac{z_{0}-\overline{z_{0}} w}{1-w}\right)=\sum_{m=0}^{\infty} c_{z_{0}}(m) w^{m} .
$$

Putting (6.3) and (6.4) together produces

$$
\sum_{m=0}^{\infty} c_{z_{0}}(m) w^{m}=\sum_{j=0}^{\infty} \frac{f^{(j)}\left(z_{0}\right)}{j !}(2 i \beta)^{j+k / 2} \frac{w^{j}}{(1-w)^{j+k}} .
$$

Use the well-known identity

$$
\frac{1}{(1-w)^{a}}=\sum_{l=0}^{\infty}\left(\begin{array}{c}
a-1+l \\
a-1
\end{array}\right) w^{l}
$$

for $a \in \mathbb{N}$ in (6.5) and compare the coefficients of $w^{m}$ to complete the proof.

Applying the proposition to $\Delta(z)$ at $z_{0}=i$ we find

$$
\begin{aligned}
c_{i}(m) & =\sum_{r=0}^{m}\left(\begin{array}{c}
11+m \\
11+r
\end{array}\right)(2 i)^{r+6} \Delta^{(r)}(i) / r ! \\
& =-2^{6} \sum_{r=0}^{m}\left(\begin{array}{c}
11+m \\
11+r
\end{array}\right) \sum_{n=1}^{\infty} \tau(n) e^{-2 \pi n}(-4 \pi n)^{r} / r ! .
\end{aligned}
$$

It is clear from (6.7) that $c_{i}(m) \in \mathbb{R}$ and from (4.6) we know that $c_{i}(m)=0$ for $m$ odd. Evaluating the $c_{i}(m)$ numerically we have $\left(\left.\Delta\right|_{12} \sigma_{i}\right)(w) \approx-0.114+1.094 w^{2}-2.621 w^{4}-6.694 w^{6}+37.787 w^{8}+O\left(w^{10}\right)$. 
With the Chowla-Selberg formula [3, p. 110] we may recognize the first term as

$$
c_{i}(0)=-(4 \pi)^{-6}\left(\frac{\Gamma(1 / 4)}{\Gamma(3 / 4)}\right)^{12} .
$$

We will return to this interesting topic in future work.

7. The dimension of $S_{k}^{n}$. The first-order space $S_{k}$ is finite-dimensional. See [25, Theorem 2.24] for an exact formula for $\operatorname{dim} S_{k}$ in terms of the signature of $\Gamma$. We begin our study of higher-order forms, in this section, by showing that $S_{k}^{n}$ being finite-dimensional follows from $\Gamma$ being finitely generated. Suppose $\Gamma \backslash \mathbb{H}$ has genus $g, r$ elliptic fixed points and $p$ cusps; then there are $2 g$ hyperbolic elements $\gamma_{i}, r$ elliptic elements $\varepsilon_{i}$ and $p$ parabolic elements $\pi_{i}$ generating $\Gamma$ and satisfying the $r+1$ relations:

$$
\left[\gamma_{1}, \gamma_{g+1}\right] \ldots\left[\gamma_{g}, \gamma_{2 g}\right] \varepsilon_{1} \ldots \varepsilon_{r} \pi_{1} \ldots \pi_{p}=1, \quad \varepsilon_{j}^{e_{j}}=1
$$

for $1 \leq j \leq r$ and integers $e_{j} \geq 2$ as in [13, Proposition 2.6]. Here $[a, b]$ denotes the commutator $a b a^{-1} b^{-1}$ of $a, b$.

Lemma 17. For every elliptic element $\varepsilon$ in $\Gamma$ and every $f$ in $S_{k}^{n}$ we have $\left.f\right|_{k}(\varepsilon-1)=0$.

Proof. This follows by induction and the fact that if $f \in S_{k}^{n}$ then $\left.f\right|_{k}(\varepsilon-1)$ $\in S_{k}^{n-1}$.

We noted in the introduction that $S_{k}^{n_{1}} \subseteq S_{k}^{n_{2}}$ for any two integers $0 \leq$ $n_{1} \leq n_{2}$. So we may consider the map

$$
\mathcal{P}_{n}: S_{k}^{n} / S_{k}^{n-1} \rightarrow\left(S_{k}^{n-1} / S_{k}^{n-2}\right)^{2 g}
$$

given by

$$
f \mapsto\left(\left.f\right|_{k}\left(\gamma_{1}-1\right),\left.f\right|_{k}\left(\gamma_{2}-1\right), \ldots,\left.f\right|_{k}\left(\gamma_{2 g}-1\right)\right)
$$

with the hyperbolic generators of (7.1).

Lemma 18. The map $\mathcal{P}_{n}$ is well defined, linear and one-to-one.

Proof. To see that $\mathcal{P}_{n}$ is well defined we note that if $f, g \in S_{k}^{n}$ represent the same element in $S_{k}^{n} / S_{k}^{n-1}$ then $f-g=h$ for $h \in S_{k}^{n-1}$. Hence $\left.f\right|_{k}\left(\gamma_{i}-1\right)$ $-\left.g\right|_{k}\left(\gamma_{i}-1\right)=\left.h\right|_{k}\left(\gamma_{i}-1\right) \in S_{k}^{n-2}$ and each component of $\mathcal{P}_{n}$ is well defined. The map $\mathcal{P}_{n}$ is clearly linear. To show it is one-to-one we examine $\operatorname{ker}\left(\mathcal{P}_{n}\right)$. If $f \in \operatorname{ker}\left(\mathcal{P}_{n}\right)$ then $\left.f\right|_{k}\left(\gamma_{i}-1\right) \in S_{k}^{n-2}$ for every hyperbolic generator $\gamma_{i}$. By definition $\left.f\right|_{k}\left(\pi_{i}-1\right)=0$ for all parabolic generators and, with Lemma $17,\left.f\right|_{k}\left(\varepsilon_{i}-1\right)=0$ for all elliptic generators. Therefore $\left.f\right|_{k}(\gamma-1) \in S_{k}^{n-2}$ for all $\gamma \in \Gamma$ and $f \in S_{k}^{n-1}$. Thus $\operatorname{ker}\left(\mathcal{P}_{n}\right)=0$ in $S_{k}^{n} / S_{k}^{n-1}$ and the map is one-to-one. 
We see then that

$$
\operatorname{dim}\left(S_{k}^{n} / S_{k}^{n-1}\right) \leq 2 g \operatorname{dim}\left(S_{k}^{n-1} / S_{k}^{n-2}\right)
$$

and

$$
\operatorname{dim}\left(S_{k}^{n} / S_{k}^{n-1}\right) \leq(2 g)^{n-1} \operatorname{dim}\left(S_{k}^{1}\right) .
$$

It follows that $S_{k}^{n} / S_{k}^{n-1}$ is finite-dimensional for all orders $n$. We may also write (7.3) as

$$
\operatorname{dim}\left(S_{k}^{n}\right) \leq \operatorname{dim}\left(S_{k}^{n-1}\right)+(2 g)^{n-1} \operatorname{dim}\left(S_{k}^{1}\right)
$$

so that

$$
\operatorname{dim}\left(S_{k}^{n}\right) \leq \frac{(2 g)^{n}-1}{2 g-1} \operatorname{dim}\left(S_{k}^{1}\right)
$$

and $S_{k}^{n}$ is also finite-dimensional. Similar arguments appear in $[17$, p. 452] and [1, Theorem 2.3].

In the case where $\Gamma$ has a parabolic element the following more precise result is demonstrated in [4]:

THEOREM 19. For $k$ in $2 \mathbb{Z}$ and $\Gamma \backslash \mathbb{H}$ non-compact with genus $g$ we have

$$
\begin{aligned}
& \operatorname{dim} S_{k}^{2}=0 \quad \text { if } k \leq 0, \\
& \operatorname{dim} S_{2}^{2}= \begin{cases}0 & \text { if } \operatorname{dim} S_{2}=0, \\
(2 g+1) \operatorname{dim} S_{2}-1 & \text { otherwise, }\end{cases} \\
& \operatorname{dim} S_{k}^{2}=(2 g+1) \operatorname{dim} S_{k} \quad \text { if } k \geq 4 .
\end{aligned}
$$

Diamantis and Sim in [5] have recently extended Theorem 19 to all higher orders. For example, the following formula may be derived from their $[5$, Theorem 4.1]. Suppose $n \in \mathbb{N}$ and $4 \leq k \in 2 \mathbb{Z}$. If $g \geq 2$ is the genus of non-compact $\Gamma \backslash \mathbb{H}$ we have

$$
\operatorname{dim} S_{k}^{n}=\left\lfloor\frac{G^{n+1}}{2(G-g)(G-1)}\right\rfloor \operatorname{dim} S_{k}
$$

when $G=g+\sqrt{g^{2}-1}$. They also handle the difficult weight 2 case. Their method of proof involves constructing non-holomorphic parabolic Poincaré series that have the desired order $n$ transformation properties and that also depend on a parameter $s \in \mathbb{C}$. The elements of $S_{k}^{n}$ are obtained by meromorphically continuing these series to $s=0$. The technical details quickly become formidable.

In the next section we show how Petersson's ideas extend smoothly into order 2. As described in the final section, we expect these results to generalize to all orders and help further our understanding of $S_{k}^{n}$. 


\section{Constructing second-order forms}

8.1. To construct higher-order Poincaré series we extend the constructions (2.6), (3.5) and (4.7) by including a map $L: \Gamma \rightarrow \mathbb{C}$ as follows. Recall the definitions $(2.5),(3.4),(4.3)$ and set

$$
\begin{aligned}
\Phi_{\mathrm{Par}}(z, m, \mathfrak{a} ; L) & :=\sum_{\gamma \in \Gamma_{\mathfrak{a}} \backslash \Gamma} L(\gamma)\left(\left.\left(A_{\mathfrak{a}}^{-1} e^{2 \pi i m \cdot}\right)\right|_{k} \gamma\right)(z), \\
\Phi_{\mathrm{Hyp}}(z, m, \eta ; L) & :=\sum_{\gamma \in \Gamma_{\eta} \backslash \Gamma} L(\gamma)\left(\left.\left(A_{\eta}^{-1} e^{2 \pi i m \cdot}\right)\right|_{k} \gamma\right)(z), \\
\Phi_{\mathrm{Ell}}\left(z, m, z_{0} ; L\right) & :=\sum_{\gamma \in \Gamma_{z_{0}} \backslash \Gamma} L(\gamma)\left(\left.\left(A_{z_{0}}^{-1} e^{2 \pi i m \cdot}\right)\right|_{k} \gamma\right)(z) .
\end{aligned}
$$

If $L$ is the constant map $L: \Gamma \rightarrow 1$, then we recover the first-order series. Let $\operatorname{Hom}(\Gamma, \mathbb{C})$ be the space of homomorphisms from $\Gamma$ to $\mathbb{C}$ and let $\operatorname{Hom}_{0}(\Gamma, \mathbb{C})$ be the subspace of maps that are zero on all parabolic elements of $\Gamma$. As we see in this section, for $L \in \operatorname{Hom}_{0}(\Gamma, \mathbb{C})$ the series $(8.1),(8.2),(8.3)$ are second-order cusp forms. (For the hyperbolic series (8.2) to be well defined we also require $L: \Gamma_{\eta} \rightarrow 0$.)

We next prove Theorem 2, on second-order relative Poincaré series.

Proof of Theorem 2. First, for $L \in \operatorname{Hom}_{0}(\Gamma, \mathbb{C})$ define $\Lambda_{L}^{+}(z)$ and $\Lambda_{L}^{-}(z)$ as follows. By a well-known theorem of Eichler and Shimura there exist unique $f^{+}, f^{-}$in $S_{2}(\Gamma)$ so that

$$
L(\gamma)=\int_{z}^{\gamma z} f^{+}(w) d w+\int_{z}^{\overline{\gamma z} f^{-}(w) d w .}
$$

The right side above is independent of $z$ and the path of integration in $\mathbb{H}$. Set

$$
\Lambda_{L}^{+}(z):=\int_{i}^{z} f^{+}(w) d w, \quad \Lambda_{L}^{-}(z):=\int_{i}^{z} f^{-}(w) d w .
$$

Then clearly, for all $z$ in $\mathbb{H}$,

$$
L(\gamma)=\Lambda_{L}^{+}(\gamma z)-\Lambda_{L}^{+}(z)+\overline{\Lambda_{L}^{-}(\gamma z)}-\overline{\Lambda_{L}^{-}(z)} .
$$

Set

$$
\begin{aligned}
P^{+}[\phi, L](z) & :=\sum_{\gamma \in \Gamma_{0} \backslash \Gamma} \Lambda_{L}^{+}(\gamma z)\left(\left.\phi\right|_{k} \gamma\right)(z), \\
P^{-}[\phi, L](z) & :=\sum_{\gamma \in \Gamma_{0} \backslash \Gamma} \overline{\Lambda_{L}^{-}(\gamma z)}\left(\left.\phi\right|_{k} \gamma\right)(z)
\end{aligned}
$$

so that

$$
P[\phi, L](z)=P^{+}[\phi, L](z)+P^{-}[\phi, L](z)-\Lambda_{L}^{+}(z) P[\phi](z)-\overline{\Lambda_{L}^{-}(z)} P[\phi](z) .
$$


Then, using (1.6) and Theorem 1 with $\phi(z)$ replaced by $\Lambda_{L}^{+}(z) \phi(z)$, we have $P^{+}[\phi, L] \in S_{k}$. The series $P^{-}[\phi, L]$ is not holomorphic but it does satisfy $\left.P^{-}[\phi, L]\right|_{k}(\gamma-1)=0$ for all $\gamma \in \Gamma$ and, using the proof of Theorem 1 and (1.6), we obtain

$$
y^{k / 2}\left|P^{-}[\phi, L](z)\right| \leq \sum_{\gamma \in \Gamma_{0} \backslash \Gamma} \operatorname{Im}(\gamma z)^{k / 2}\left|\Lambda_{L}^{-}(\gamma z) \phi(\gamma z)\right|<\infty .
$$

Now $f \in S_{k}$ implies $y^{k / 2}|f(z)| \ll 1$ uniformly for all $z \in \mathbb{H}$. Therefore

$$
\begin{aligned}
y^{k / 2}|P[\phi, L](z)| \leq & y^{k / 2}\left|P^{+}[\phi, L](z)\right|+y^{k / 2}\left|P^{-}[\phi, L](z)\right| \\
& +y^{k / 2}\left|\Lambda_{L}^{+}(z) P[\phi](z)\right|+y^{k / 2}\left|\Lambda_{L}^{-}(z) P[\phi](z)\right| \\
\ll & 1+\left|\Lambda_{L}^{+}(z)\right|+\left|\Lambda_{L}^{-}(z)\right| .
\end{aligned}
$$

Let $\mathfrak{F}$ be a fixed fundamental domain for $\Gamma \backslash \mathbb{H}$ intersecting $\mathbb{R} \cup \infty$ at a finite number of cusps. For each such cusp $\mathfrak{a}$ the scaling matrix $\sigma_{\mathfrak{a}}$ maps $z \in \mathbb{H}$ with $0 \leq x<1$ and $y$ large into a neighborhood of $\mathfrak{a}$, as in Figure 1. Since $f^{+}, f^{-}$are bounded on $\mathfrak{F}$ and have exponential decay at cusps, we must have $\left|\Lambda_{L}^{+}(z)\right|,\left|\Lambda_{L}^{-}(z)\right| \ll 1$ on $\mathfrak{F}$ so that (8.6) implies

$$
y^{k / 2}|P[\phi, L](z)| \ll 1 \quad \text { for } z \in \mathfrak{F} .
$$

It follows that $P[\phi, L](z)$ converges absolutely and uniformly on all compact sets of $\mathbb{H}$ to a holomorphic function. It is easy to check that

$$
\left.P[\phi, L]\right|_{k}(\gamma-1)=-L(\gamma) P[\phi] \in S_{k}^{1}(\Gamma)
$$

for all $\gamma \in \Gamma$. Since $L \in \operatorname{Hom}_{0}(\Gamma, \mathbb{C})$ we also have $\left.P[\phi, L]\right|_{k}(\pi-1)=0$ for all parabolic $\pi \in \Gamma$.

It only remains to check that $P[\phi, L]$ has rapid decay at each cusp. At any cusp $\mathfrak{a}$ of $\mathfrak{F}$ we have a Fourier expansion

$$
j\left(\sigma_{\mathfrak{a}}, z\right)^{-k} P[\phi, L]\left(\sigma_{\mathfrak{a}} z\right)=\sum_{m \in \mathbb{Z}} b_{\mathfrak{a}}(m) e^{2 \pi i m z}
$$

but

$$
\left|j\left(\sigma_{\mathfrak{a}}, z\right)^{-k} P[\phi, L]\left(\sigma_{\mathfrak{a}} z\right)\right| \leq y^{-k / 2}\left|\operatorname{Im}\left(\sigma_{\mathfrak{a}} z\right)^{k / 2} P[\phi, L]\left(\sigma_{\mathfrak{a}} z\right)\right| \ll y^{-k / 2}
$$

for $z$ with $0 \leq x<1$ and $y$ large. Consequently, $j\left(\sigma_{\mathfrak{a}}, z\right)^{-k} P[\phi, L]\left(\sigma_{\mathfrak{a}} z\right) \rightarrow 0$ as $y \rightarrow \infty$ uniformly for $0 \leq x<1$. Hence we must have $b_{\mathfrak{a}}(m)=0$ in (8.8) for all $m \leq 0$ and $P[\phi, L]$ has rapid decay at the cusp $\mathfrak{a}$. By the discussion at the end of Section 2.1 it is enough to verify the rapid decay condition at the cusps of $\mathfrak{F}$.

REMARK. We note that the bound (8.7) is not true in general if we allow $z \in \mathbb{H}$. For example, setting $U(z)=y^{k / 2}|P[\phi, L](z)|$ we see that

$$
U\left(\gamma^{m} z\right)=y^{k / 2}|P[\phi, L](z)-m L(\gamma) P[\phi](z)|
$$

becomes unbounded as $m \rightarrow \infty$ if $L(\gamma)$ and $P[\phi](z)$ are non-zero. 
8.2. Now we have everything in place to verify our constructions.

Theorem 20. For $4 \leq k \in 2 \mathbb{Z}$ and $L \in \operatorname{Hom}_{0}(\Gamma, \mathbb{C})$ we have

$$
\begin{aligned}
\Phi_{\mathrm{Par}}(z, m, \mathfrak{a} ; L) \in S_{k}^{2} & \text { for } m \in \mathbb{N}, \\
\Phi_{\mathrm{Hyp}}(z, m, \eta ; L) \in S_{k}^{2} & \text { for } m \in \mathbb{Z}, L\left(\gamma_{\eta}\right)=0, \\
\Phi_{\mathrm{Ell}}\left(z, m, z_{0} ; L\right) \in S_{k}^{2} & \text { for } m \in \mathbb{N}, N m-k / 2 \geq 0,
\end{aligned}
$$

where $N$ in (8.11) is $\left|\Gamma_{z_{0}}\right|$, the order of the subgroup of elements fixing $z_{0}$.

The second-order parabolic series $\Phi_{\mathrm{Par}}(z, m, \mathfrak{a} ; L)$ appears first, for $m=0$ and $\Gamma=\mathrm{PSL}_{2}(\mathbb{Z})$, in Section 4 of Eichler's paper [6]. In fact, he allows $L$ to be any period polynomial (the degree 0 polynomials correspond to $\left.\operatorname{Hom}_{0}(\Gamma, \mathbb{C})\right)$ and uses the second-order parabolic series to prove the existence of general abelian integrals with prescribed periods. The general result (8.9) is shown, with a different proof to the one given here, in [4, Prop. 4.2].

Proof of Theorem 20. The parabolic series. We proceed as in Proposition 4 , beginning with $\Phi_{\operatorname{Par}}(z, m, \mathfrak{a} ; L)$. According to Theorem 2 we need to verify the condition

$$
\int_{0}^{\infty} \int_{0}^{1}\left(1+\left|\Lambda_{L}^{+}\left(\sigma_{\mathfrak{a}} z\right)\right|+\left|\Lambda_{L}^{-}\left(\sigma_{\mathfrak{a}} z\right)\right|\right) e^{-2 \pi m y} y^{k / 2-2} d x d y<\infty .
$$

The estimates

$$
\Lambda_{L}^{+}\left(\sigma_{\mathfrak{a}} z\right), \Lambda_{L}^{-}\left(\sigma_{\mathfrak{a}} z\right) \ll 1+|\log y|,
$$

when $|\operatorname{Re}(z)| \leq 1$, say, with an implied constant depending only on $L, \sigma_{\mathfrak{a}}$ and $\Gamma$, follow from noting that for any $f \in S_{2}(\Gamma)$ (or $f \in S_{2}\left(\sigma_{\mathfrak{a}}^{-1} \Gamma \sigma_{\mathfrak{a}}\right)$ ) we know that $y|f(z)|$ is bounded for $z \in \mathbb{H}[12$, p. 70]. Thus, the left side of (8.12) is less than

$$
\int_{0}^{\infty}(1+|\log y|) e^{-2 \pi m y} y^{k / 2-2} d y .
$$

We leave it to the reader to show that this is bounded for $k>2$ and $m \in \mathbb{N}$.

The hyperbolic series. For the series $\Phi_{\mathrm{Hyp}}(z, m, \eta ; L)$ we must check that

$$
\begin{aligned}
\int_{1}^{\mu} \int_{0}^{\pi}\left(1+\left|\Lambda_{L}^{+}\left(\sigma_{\eta}\left(r e^{i \theta}\right)\right)\right|+\right. & \left.\left|\Lambda_{L}^{-}\left(\sigma_{\eta}\left(r e^{i \theta}\right)\right)\right|\right) \\
& \times r^{-1} e^{-\pi m \theta / \log \xi}(\sin \theta)^{k / 2-2} d \theta d r<\infty
\end{aligned}
$$

is satisfied. The bounds

$$
\Lambda_{L}^{+}\left(\sigma_{\eta} z\right), \Lambda_{L}^{-}\left(\sigma_{\eta} z\right) \ll 1+|\log y|,
$$

with an implied constant depending only on $L, \sigma_{\mathfrak{a}}$ and $\Gamma$, are proved in the same way as (8.13). With these the reader may confirm that (8.14) is true for $k>2$ and $m \in \mathbb{Z}$. 
The elliptic series. For the series $\Phi_{\text {Ell }}\left(z, m, z_{0} ; L\right)$ we need

$$
\begin{aligned}
\int_{0}^{1} \int_{\pi-\pi / N}^{\pi+\pi / N}\left(1+\left|\Lambda_{L}^{+}\left(\sigma_{z_{0}}\left(r e^{i \theta}\right)\right)\right|+\right. & \left.\left|\Lambda_{L}^{-}\left(\sigma_{z_{0}}\left(r e^{i \theta}\right)\right)\right|\right) \\
& \times r^{m+1}\left(1-r^{2}\right)^{k / 2-2} d \theta d r<\infty .
\end{aligned}
$$

As we saw in (4.1) and Figure 3, $\sigma_{z_{0}}\left(r e^{i \theta}\right)$ will be contained in a bounded region of $\mathbb{H}$. Thus we find

$$
\Lambda_{L}^{+}\left(\sigma_{z_{0}}\left(r e^{i \theta}\right)\right) \ll 1+\left|\log \operatorname{Im}\left(\sigma_{z_{0}}\left(r e^{i \theta}\right)\right)\right| .
$$

Similarly, for $\Lambda_{L}^{-}$and using (4.9), the left side of (8.15) is bounded by a finite constant times

$$
\int_{0}^{1} \int_{\pi-\pi / N}^{\pi+\pi / N}\left(1+\left|\log \frac{1-r^{2}}{\left|1-r e^{i \theta}\right|^{2}}\right|\right) r^{m+1}\left(1-r^{2}\right)^{k / 2-2} d \theta d r .
$$

We have

$$
\left|\log \frac{1-r^{2}}{\left|1-r e^{i \theta}\right|^{2}}\right| \leq\left|\log \left(1-r^{2}\right)\right|+2|\log | 1-r e^{i \theta}||
$$

and since $N \geq 2$ we know

$$
|\log | 1-r e^{i \theta}|| \leq \log 2 .
$$

Consequently, (8.16) is bounded by a constant times

$$
\int_{0}^{1}\left(1+\left|\log \left(1-r^{2}\right)\right|\right) r^{m+1}\left(1-r^{2}\right)^{k / 2-2} d r .
$$

It is straightforward to verify that this is bounded for $k>2$ and $m \in \mathbb{N}_{0}$.

\section{Spanning questions}

9.1. If $\Gamma$ contains a parabolic element, or equivalently if $\Gamma \backslash \mathbb{H}$ is noncompact with a cusp $\mathfrak{a}$, then let

$$
\mathcal{S}_{\text {Par }}^{1}(\mathfrak{a}):=\left\{\Phi_{\text {Par }}(z, m, \mathfrak{a}): m \in \mathbb{N}\right\}
$$

be the set of all weight $k$ parabolic Poincare series associated to this cusp. The elements in this set span the weight $k$ cusp forms

$$
S_{k}=\left\langle\mathcal{S}_{\text {Par }}^{1}(\mathfrak{a})\right\rangle
$$

for the simple reason that any element in a subspace orthogonal to all of $\mathcal{S}_{\text {Par }}^{1}(\mathfrak{a})$ must have an identically zero parabolic expansion at $\mathfrak{a}$ by Proposition 5 .

If $\Gamma$ does not contain parabolic elements then we cannot construct parabolic Poincaré series. If $\Gamma$ has an elliptic fixed point $z_{0}$ then similar reasoning 
with Proposition 15 shows that the weight $k$ elliptic Poincaré series

$$
\mathcal{S}_{\mathrm{Ell}}^{1}\left(z_{0}\right):=\left\{\Phi_{\mathrm{Ell}}\left(z, m, z_{0}\right): m \in \mathbb{N}_{0}\right\}
$$

$\operatorname{span} S_{k}$.

It is possible that $\Gamma$ does not contain any parabolic or elliptic elements. It must contain a hyperbolic element though, by [16, Theorem 2.4.4]. If $\eta=\left\{\eta_{1}, \eta_{2}\right\}$ is any pair of hyperbolic fixed points then, with Proposition 8 , $S_{k}$ is spanned by the weight $k$ hyperbolic Poincaré series

$$
\mathcal{S}_{\mathrm{Hyp}}^{1}(\eta):=\left\{\Phi_{\mathrm{Hyp}}(z, m, \eta): m \in \mathbb{Z}\right\} .
$$

9.2. We would expect the weight $k$ second-order Poincaré series to span the space $S_{k}^{2} / S_{k}^{1}$ and this is indeed the case, provided we allow the homomorphisms $L$ as well as the indices $m$ to vary. Similarly to the first-order sets in Section 9.1, define

$$
\begin{aligned}
& \mathcal{S}_{\text {Par }}^{2}(\mathfrak{a}):=\left\{\Phi_{\text {Par }}(z, m, \mathfrak{a} ; L): m \in \mathbb{N}, L \in \operatorname{Hom}_{0}(\Gamma, \mathbb{C})\right\}, \\
& \mathcal{S}_{\text {Ell }}^{2}\left(z_{0}\right):=\left\{\Phi_{\text {Ell }}\left(z, m, z_{0} ; L\right): m \in \mathbb{N}_{0}, L \in \operatorname{Hom}_{0}(\Gamma, \mathbb{C})\right\} .
\end{aligned}
$$

Recall the hyperbolic generators (7.1) for $\Gamma$. For $1 \leq i \leq 2 g$ define the homomorphisms $L_{\gamma_{i}} \in \operatorname{Hom}_{0}(\Gamma, \mathbb{C})$ dual to these generators by

$$
L_{\gamma_{i}}\left(\gamma_{j}\right):=\delta_{i j}
$$

and each $L_{\gamma_{i}}$ is zero on the other elliptic and parabolic generators. It follows that $\left\{L_{\gamma_{i}}: 1 \leq i \leq 2 g\right\}$ is a basis for $\operatorname{Hom}_{0}(\Gamma, \mathbb{C})$.

Proposition 21. For a cusp $\mathfrak{a}$ of $\Gamma \backslash \mathbb{H}$ or an elliptic fixed point $z_{0}$, the sets $\mathcal{S}_{\mathrm{Par}}^{2}(\mathfrak{a}), \mathcal{S}_{\mathrm{Ell}}^{2}\left(z_{0}\right)$ each span $S_{k}^{2} / S_{k}^{1}$.

Proof. Recall the map $\mathcal{P}_{2}: S_{k}^{2} / S_{k}^{1} \rightarrow\left(S_{k}^{1}\right)^{2 g}$ given by (7.2). A short calculation shows that

$$
\frac{\Phi_{\operatorname{Par}}\left(\gamma_{j} z, m, \mathfrak{a} ; L_{\gamma_{i}}\right)}{j\left(\gamma_{j}, z\right)^{k}}=\Phi_{\operatorname{Par}}\left(z, m, \mathfrak{a} ; L_{\gamma_{i}}\right)-L_{\gamma_{i}}\left(\gamma_{j}\right) \Phi_{\operatorname{Par}}(z, m, \mathfrak{a}) .
$$

Hence

$$
\mathcal{P}_{2}\left(\Phi_{\operatorname{Par}}\left(z, m, \mathfrak{a} ; L_{\gamma_{i}}\right)\right)=(0,0, \ldots, \underbrace{-\Phi_{\text {Par }}(z, m, \mathfrak{a})}_{i \text { th component }}, \ldots, 0) .
$$

Let $F$ be any element of $S_{k}^{2}$ and

$$
\mathcal{P}_{2}(F)=\left(f_{1}, f_{2}, \ldots, f_{2 g}\right) .
$$

Since we know that the $\Phi_{\operatorname{Par}}(z, m, \mathfrak{a})$ span $S_{k}$, we may write each $f_{i}$ as a finite sum

$$
f_{i}(z)=\sum_{m} c_{i}(m) \Phi_{\operatorname{Par}}(z, m, \mathfrak{a}) .
$$


Therefore

$$
\mathcal{P}_{2}\left(F(z)+\sum_{i=1}^{2 g} \sum_{m} c_{i}(m) \Phi_{\operatorname{Par}}\left(z, m, \mathfrak{a} ; L_{\gamma_{i}}\right)\right)=0,
$$

from which it follows that

$$
F(z)+\sum_{i=1}^{2 g} \sum_{m} c_{i}(m) \Phi_{\operatorname{Par}}\left(z, m, \mathfrak{a} ; L_{\gamma_{i}}\right) \in S_{k}^{1}
$$

and $S_{k}^{2} / S_{k}^{1}$ is spanned by $\mathcal{S}_{\text {Par }}^{2}(\mathfrak{a})$ as we wanted to show. The proof for $\mathcal{S}_{\text {Ell }}^{2}\left(z_{0}\right)$ is identical.

The hyperbolic version of this result is slightly more involved in that, as well as $L$ and $m$, we also need to vary the hyperbolic points $\eta$. For a simple choice, put

$$
\begin{aligned}
\mathcal{A} & :=\left\{\Phi_{\text {Hyp }}\left(z, m, \eta\left(\gamma_{1}\right) ; L_{\gamma_{i}}\right): m \in \mathbb{Z}, 2 \leq i \leq 2 g\right\}, \\
\mathcal{B} & :=\left\{\Phi_{\text {Hyp }}\left(z, m, \eta\left(\gamma_{2}\right) ; L_{\gamma_{1}}\right): m \in \mathbb{Z}\right\} .
\end{aligned}
$$

Proposition 22. The set $\mathcal{S}_{\mathrm{Hyp}}^{2}=\mathcal{A} \cup \mathcal{B}$ spans $S_{k}^{2} / S_{k}^{1}$.

Proof. With similar reasoning to the proof of Proposition 21 we see that $\mathcal{A}$ spans the subspace of $S_{k}^{2} / S_{k}^{1}$ with image $\left(0, f_{2}, f_{3}, \ldots, f_{2 g}\right)$ under $\mathcal{P}_{2}$, and $\mathcal{B}$ spans the subspace with image $\left(f_{1}, 0, \ldots, 0\right)$, where the $f_{i}$ are arbitrary elements of $S_{k}^{1}$. Therefore, as in Proposition 21, for any $F \in S_{k}^{2}$ there exists $G$ in the space spanned by $\mathcal{A} \cup \mathcal{B}$ with $\mathcal{P}_{2}(F+G)=0$. Consequently, $F+G \in S_{k}^{1}$ and $S_{k}^{2} / S_{k}^{1}$ is spanned by $\mathcal{A} \cup \mathcal{B}$.

REMARK. We saw in Section 3.3 that the set

$$
\mathcal{T}_{\mathrm{Hyp}}^{1}:=\left\{\Phi_{\mathrm{Hyp}}(z, 0, \eta(\gamma)): \gamma \in \operatorname{Hyp}(\Gamma)\right\}
$$

also spans $S_{k}$. The obvious second-order analog is

$$
\mathcal{T}_{\text {Hyp }}^{2}:=\left\{\Phi_{\text {Hyp }}(z, 0, \eta(\gamma) ; L): \gamma \in \operatorname{Hyp}(\Gamma), L \in \operatorname{Hom}_{0}(\Gamma, \mathbb{C}), L(\gamma)=0\right\} .
$$

We expect that this set spans $S_{k}^{2} / S_{k}^{1}$, but have been unable to prove it.

The proof that the first-order Poincaré series span $S_{k}$ relies on Petersson's inner product (2.8) and the fact that each type of these seriesparabolic, hyperbolic and elliptic - pick out corresponding expansion coefficients, as we saw in Propositions 5, 8 and 15. Is there a similar proof that second-order Poincaré series span $S_{k}^{2}$ ? The answer is yes, but the inner product on $S_{k}^{2}$ is not given by Petersson's formula (2.8). See [11] for the details.

10. Higher-order forms. We saw in Section 8 that the usual Poincaré series in $S_{k}^{1}$ could be generalized to series in $S_{k}^{2}$ by including $L \in \operatorname{Hom}(\Gamma, \mathbb{C})$ in their definition. Together these series spanned $S_{k}^{2}$. What plays the role of $L$ when we look to construct third-order and higher-order forms? 
First, we generalize our "" notation slightly. For any $L: \Gamma \rightarrow \mathbb{C}$ and $\gamma \in$ $\Gamma$ define $L \mid \gamma:=L(\gamma)$ and extend this linearly to $\mathbb{C}\left[\mathrm{SL}_{2}(\mathbb{R})\right]$ and $\mathbb{C}\left[\mathrm{PSL}_{2}(\mathbb{R})\right]$. Consider the functions $L_{1}, L_{2}: \Gamma \rightarrow \mathbb{C}$ satisfying

$$
\begin{aligned}
L_{1} \mid\left(\delta_{1}-1\right) & =0 & & \text { for all } \delta_{1} \in \Gamma, \\
L_{2} \mid\left(\delta_{1}-1\right)\left(\delta_{2}-1\right) & =0 & & \text { for all } \delta_{1}, \delta_{2} \in \Gamma,
\end{aligned}
$$

and in general, for $n \in \mathbb{N}$, let $\operatorname{Hom}^{[n]}(\Gamma, \mathbb{C})$ be the space of all functions $L_{n}: \Gamma \rightarrow \mathbb{C}$ where

$$
L_{n} \mid\left(\left(\delta_{1}-1\right) \cdots\left(\delta_{n}-1\right)\right)=0 \quad \text { for all } \delta_{1}, \ldots, \delta_{n} \in \Gamma \text {. }
$$

Clearly, $\operatorname{Hom}^{[1]}(\Gamma, \mathbb{C})$ is just the space of constant functions, and is isomorphic to $\mathbb{C}$. The functions $L_{2} \in \operatorname{Hom}^{[2]}(\Gamma, \mathbb{C})$ satisfy

$$
L_{2}\left(\delta_{1} \delta_{2}\right)=L_{2}\left(\delta_{1}\right)+L_{2}\left(\delta_{2}\right)-L_{2}(I) \quad \text { for all } \delta_{1}, \delta_{2} \in \Gamma
$$

and we have $L_{2}-L_{2}(I) \in \operatorname{Hom}(\Gamma, \mathbb{C})$. Hence

$$
\operatorname{Hom}^{[2]}(\Gamma, \mathbb{C}) \cong \mathbb{C} \oplus \operatorname{Hom}(\Gamma, \mathbb{C}) .
$$

Similarly, if we set

$$
\begin{aligned}
& \operatorname{Hom}_{0}^{[n]}(\Gamma, \mathbb{C}):=\left\{L_{n} \in \operatorname{Hom}^{[n]}(\Gamma, \mathbb{C}):\right. \\
& \left.\qquad L_{n} \mid(\pi-1)=0 \text { for all parabolic } \pi \in \Gamma\right\}
\end{aligned}
$$

then

$$
\operatorname{Hom}_{0}^{[2]}(\Gamma, \mathbb{C}) \cong \mathbb{C} \oplus \operatorname{Hom}_{0}(\Gamma, \mathbb{C}) .
$$

Recall we have shown that the full second-order space is spanned by Poincaré series. For example, with (9.1) and Proposition 21 we have

$$
S_{k}^{2}=\left\langle\mathcal{S}_{\text {Par }}^{1}(\mathfrak{a}) \cup \mathcal{S}_{\text {Par }}^{2}(\mathfrak{a})\right\rangle
$$

in the parabolic case and similarly for the elliptic and hyperbolic series. Thus, by (10.1) we have

$$
S_{k}^{2}=\left\langle\left\{\Phi_{\text {Par }}(z, m, \mathfrak{a} ; L): m \in \mathbb{N}, L \in \operatorname{Hom}_{0}^{[2]}(\Gamma, \mathbb{C})\right\}\right\rangle .
$$

Looking towards future work, we expect that, for all $n \in \mathbb{N}$,

$$
S_{k}^{n}=\left\langle\left\{\Phi_{\text {Par }}(z, m, \mathfrak{a} ; L): m \in \mathbb{N}, L \in \operatorname{Hom}_{0}^{[n]}(\Gamma, \mathbb{C})\right\}\right\rangle
$$

for a group with a cusp $\mathfrak{a}$, with similar results for the hyperbolic and elliptic cases. This approach should parallel that of Diamantis and Sim [5] and also be valid in the compact case.

\section{References}

[1] G. Chinta, N. Diamantis, and C. O'Sullivan, Second order modular forms, Acta Arith. 103 (2002), 209-223. 
[2] G. Chinta and D. Goldfeld, Grössencharakter L-functions of real quadratic fields twisted by modular symbols, Invent. Math. 144 (2001), 435-449.

[3] S. Chowla and A. Selberg, On Epstein's zeta-function, J. Reine Angew. Math. 227 (1967), 86-110.

[4] N. Diamantis and C. O'Sullivan, The dimensions of spaces of holomorphic secondorder automorphic forms and their cohomology, Trans. Amer. Math. Soc. 360 (2008), 5629-5666.

[5] N. Diamantis and D. Sim, The classification of higher-order cusp forms, J. Reine Angew. Math. 622 (2008), 121-153.

[6] M. Eichler, Grenzkreisgruppen und kettenbruchartige Algorithmen, Acta Arith. 11 (1965), 169-180.

[7] D. W. Farmer and K. Wilson, Converse theorems assuming a partial Euler product, Ramanujan J. 15 (2008), 205-218.

[8] D. Goldfeld, The distribution of modular symbols, in: Number Theory in Progress (Zakopane-Kościelisko, 1997), Vol. 2, de Gruyter, Berlin, 1999, 849-865.

[9] W. Goldman and J. Millson, Eichler-Shimura homology and the finite generation of cusp forms by hyperbolic Poincaré series, Duke Math. J. 53 (1986), 1081-1091.

[10] T. Hiramatsu, Eichler maps and hyperbolic Fourier expansion, Nagoya Math. J. 40 (1970), 173-192.

[11] Ö. Imamoḡlu and C. O'Sullivan, Higher-order cusp forms: inner products, in preparation, 2009.

[12] H. Iwaniec, Topics in Classical Automorphic Forms, Grad. Stud. Math. 17, Amer. Math. Soc., Providence, RI, 1997.

[13] —, Spectral Methods of Automorphic Forms, 2nd ed., Grad. Stud. Math. 53, Amer. Math. Soc., Providence, RI, 2002.

[14] J. Jorgenson and C. O'Sullivan, Unipotent vector bundles and higher-order nonholomorphic Eisenstein series, J. Théor. Nombres Bordeaux 20 (2008), 131-163.

[15] S. Katok, Closed geodesics, periods and arithmetic of modular forms, Invent. Math. 80 (1985), 469-480.

[16] —, Fuchsian Groups, Chicago Lectures in Math., Univ. of Chicago Press, Chicago, IL, 1992.

[17] P. Kleban and D. Zagier, Crossing probabilities and modular forms, J. Statist. Phys. 113 (2003), 431-454.

[18] M. Knopp and G. Mason, Vector-valued modular forms and Poincaré series, Illinois J. Math. 48 (2004), 1345-1366.

[19] W. Kohnen and D. Zagier, Modular forms with rational periods, in: R. A. Rankin (ed.), Modular Forms, Ellis Horwood, Chichester, 1984, 197-249.

[20] S. L. Krushkal, Quasiconformal Mappings and Riemann Surfaces, Winston, Washington, DC, 1979.

[21] M. H. Lee, Eisenstein series and Poincaré series for mixed automorphic forms, Collect. Math. 51 (2000), 225-236.

[22] G. Mason, 2-dimensional vector-valued modular forms, Ramanujan J. 17 (2008), 405-427.

[23] H. Petersson, Über eine Metrisierung der ganzen Modulformen, Jahresber. Deutsch. Math.-Ver. 49 (1939), 49-75.

[24] —, Einheitliche Begründung der Vollständigkeitssätze für die Poincaréschen Reihen von reeller Dimension bei beliebigen Grenzkreisgruppen von erster Art, Abh. Math. Sem. Hansische Univ. 14 (1941), 22-60.

[25] G. Shimura, Introduction to the Arithmetic Theory of Automorphic Functions, Princeton Univ. Press, Princeton, NJ, 1971. 
[26] C. L. Siegel, Lectures on Advanced Analytic Number Theory, Tata Inst., Bombay, 1961.

[27] D. Zagier, Modular forms associated to real quadratic fields, Invent. Math. 30 (1975), $1-46$.

Department of Mathematics

Department of Mathematics

ETH Zurich

The CUNY Graduate Center

CH-8092, Zürich, Switzerland

E-mail: ozlem@math.ethz.ch

New York, NY 10016-4309, U.S.A.

E-mail: cosullivan@gc.cuny.edu

Received on 26.10.2007

and in revised form on 15.3.2009 